\title{
Evolution, gene expression profiling and 3D modeling of CSLD proteins in cotton
}

Yanpeng $\mathrm{Li}^{1,2^{*}}$, Tiegang Yang ${ }^{1,2^{*}}$, Dandan Dai ${ }^{1,2}$, Ying $\mathrm{Hu}^{1,2}$, Xiaoyang Guo ${ }^{1,2}$ and Hongxia Guo ${ }^{1,2}$

\begin{abstract}
Background: Among CESA-like gene superfamily, the cellulose synthase-like D (CSLD) genes are most similar to cellulose synthase genes and have been reported to be involved in tip-growing cell and stem development. However, there has been no genome-wide characterization of this gene subfamily in cotton. We thus sought to analyze the evolution and functional characterization of CSLD proteins in cotton based on fully sequenced cotton genomes.

Results: A total of 23 full-length CSLD proteins were identified in Gossypium raimondii, Gossypium arboreum and Gossypium hirsutum. The phylogenetic tree divided the CSLD proteins into five clades with strong support: CSLD1, CSLD2/3, CSLD4, CSLD5 and CSLD6. The total expression of GhCSLD genes was the highest in androecium \& gynoecium (mostly contributed by CSLD1 and CSLD4) compared with other CSL genes. CSLD1 and CSLD4 were only highly expressed in androecium \& gynoecium (A\&G), and showed tissue-specific expression. The total expression of CSLD2/3, 5 and 6 was highest in the specific tissues. These results suggest that CSLD genes showed the different pattern of expression. Cotton CSLD proteins were subjected to different evolutionary pressures, and the CSLD1 and CSLD4 proteins exhibited episodic and long-term shift positive selection. The predicted three-dimensional structure of GrCSLD1 suggested that GrCSLD1 belongs to glycosyltransferase family 2. The amino acid residues under positive selection in the CSLD1 lineage are positioned in a region adjacent to the class-specific region (CSR), $\beta 1$-strand and transmembrane helices (TMHs) in the GrCSLD1structure.
\end{abstract}

Conclusion: Our results characterized the CSLD proteins by an integrated approach containing phylogeny, transcriptional profiling and 3D modeling. The study added to the understanding about the importance of the CSLD family and provide a useful reference for selecting candidate genes and their associations with the biosynthesis of the cell wall in cotton.

Keywords: Cotton, CSLD, Phylogenetic tree, Positive selection, CSL superfamily, Structural modeling, Cellulose synthase, Cell wall

\section{Background}

The plant cell wall plays a central role in plant development and is primarily composed of three polysaccharides: cellulose, hemicellulose and pectin [1-3]. Lignin is a major polymer of secondary cell wall [4]. Cellulose comprises unbranched homopolymers of $\beta$-1,4-linked glucose units and is a core structural component of the plant cell wall [5]. The biosynthesis of cellulose has attracted great interest because cellulose microfibrils are key determinants of the physical characteristics of the

\footnotetext{
*Correspondence: liyanpeng1979@163.com; ytgha@163.com ${ }^{1}$ Industrial Crop Research Institute, Henan Academy of Agricultural Sciences, No. 116, Huayuan Road, Zhengzhou 450002, China

Full list of author information is available at the end of the article
}

cell wall [6]; provide renewable resources for biofuels [7, 8]. Cellulose is synthesized by cellulose synthase (CESA) which belongs to glycosyltransferase family 2 (GT2) [9]. The CSLs, which are grouped into 10 families (CSLA, B, C, D, E, F, G, H, J, and K), and CESA form the CESA superfamily [10-12]. However, there are six families (CSLA, C, D, E, F and H) in rice [13, 14]. The CSLs are also members of GT2 [9]. In plants, CESA genes were first identified in cotton fiber based on sequence homology to bacterial CESA genes [15]. The nearly complete genome sequence of the Arabidopsis thaliana revealed 10 CESA genes $[2,12]$, which are classified as required for primary (CESA1, 2, 3, 5, 6, 9) and secondary (CESA4, $7,8)$ cell wall synthesis [16-18]. CESA1, CESA3 and 
CESA6 are considered parts of the primary wall CESA complex, and CESA 5 and CESA2 are partially functionally redundant with CESA6 at different stages of growth [16]. CESA6-related CESA9 exhibits functional redundancy with CESA6 [17]. The secondary wall CESA complex comprises CESA4, CESA7 and CESA8, as identified in irx (irregular xylem) mutants of A. thaliana [18]. In contrast to the primary wall CESA complex, these three CESA genes appear equally important for cellulose synthesis in the secondary cell wall, indicating that they are not redundant with one another [18]. In cotton, CESA1, 2, 7, 8 (the orthologs of $A$. thaliana CESA8, 4, 7 and 7, respectively) are associated in the cellulose biosynthesis secondary cell wall, whereas CESA3, 5, 6, 9 and 10 (the orthologs of $A$. thaliana CESA3, 2/5/6/9, 1/10, 2/5/6/9 and 3 , respectively) participate in primary cell wall synthesis in cotton fiber [19-22]. Moreover, CESA8 (ATCESA7) could paly an enhancer role for rapid and massive cellulose accumulation of secondary cell wall in cotton fiber development, which is quite different from other grass species [19]. More recently, it has been reported that there is a "relay race" model for fiber development involving the CesA genes in G. barbadense [23].

CSL genes encoding processive glycosyltransferases have been indicated in the biosynthesis of non-cellulosic polysaccharides in the plant wall. For instance, CSLA genes encode mannan synthases [24, 25], CSLC genes encode $\beta-1,4$ glucan synthases that mediate xyloglucan biosynthesis [26], and the CSLF and CSLH proteins are involved in $(1,3 ; 1,4)-\beta$-D-glucan biosynthesis [27, 28]. Among CSL gene families, the CSLD gene family is most similar to the CESA family and possesses the most ancient intron/exon structure [12]. The CSLDs have been implicated in cellulose and mannan synthesis [29-31]. In $A$. thaliana, mutants in five CSLD genes have been described to cause distinct phenotypes. In CSLD3 mutants, root hairs form bulges soon after initiation [32, 33], and CSLD3 is involved in the synthesis of $\beta$-1,4-glucan polysaccharide in the apical plasma membrane of root hair cells [29]. CSLD2 mutants grow abnormal root hairs [34], and there may be partial divergence and redundancy in CSLD2 and CSLD3 gene function during root hair and female gametophyte development [34, 35]. CSLD5 mutants have significantly reduced stem and root growth [36]. A recent report showed that CSLD5 participates in the construction of newly forming cell plates and is an unstable protein that is degraded upon completion of cell division [37]. Furthermore, the cooperative activities of CSLD2, CSLD3 and CSLD5 are necessary for normal development [31]. CSLD1 and CSLD4 mutants exhibit a significant reduction of cellulose deposition on pollen tubes and distinct disorder of pollen tube wall layers, suggesting that the CSLD1 and CSLD4 genes are required for normal pollen tube growth [30,34]. In rice, mutations in
OsCSLD1 and OsCSLD4, the orthologs of A. thaliana CSLD2/3 and CSLD5, respectively, have an important influence on leaf morphogenesis and plant architecture [38-41]. OsCSLD1 mutants exhibit abnormal root hair [38]. Maize CSLD1 (the ortholog of A. thaliana CSLD5) is required for cell division, expansion and leaf growth [42]. The major studies mentioned above indicate that CSLD proteins may be involved in cellulose synthesis in tip-growing cells (pollen tubes and root hairs) and stem growth.

Cotton is one of the most economically important crops, and its fiber is the main natural source for the textile industry [43]. Cotton is also an excellent model system for the study of polyploidization, cell wall biosynthesis and cell elongation [44-46]. Despite outstanding progress in A. thaliana and rice, little is known regarding CSLD proteins in cotton. The cotton CSL genes are involved in the synthesis of cell wall matrix polysaccharides that surround cellulose microfibrils in cotton [20]. The genes CSLD2/3 and CSLD6 but not CSLD1 and CSLD4 are expressed strongly in fiber development $[21,45]$. The CSLD2/3 genes have also been suggested to be involved in mannan synthesis during cotton fiber cell development [47].

The recently assembled and published genome sequences for Gossypium raimondii [20], Gossypium arboretum [48] and Gossypium hirsutum [45] provide an opportunity to identify and analyze the CSL gene family at the wholegenome level. Here, to gain insight into the evolution and functional characterization of CSLD proteins based on the cotton genome, we identified the CSLD proteins and constructed maximum likelihood (ML) and Bayesian phylogenetic trees to reconstruct the evolutionary origin of the CSLD genes. Then, gene expression, qRT-PCR and positive selection were analyzed. Finally, we generated a model of the three-dimensional structure of CSLD1 to elucidate the function of CSLD1. We show that 1) the 23 full-length CSLD proteins are divided into five clades; 2) CSLD genes show the different expression patterns compared with CESA and other CSL genes; 3) the CSLD1 and CSLD4 clades exhibit episodic and long-term shift positive selection; 4) the GrCSLD1 protein belongs to glycosyltransferase family 2 and probably participates in the biosynthesis of cellulose, mannan or other polysaccharides. These results provide a thorough picture of the evolution and biological and molecular function of CSLD proteins in cotton.

\section{Results}

\section{Distribution of CSLD proteins in the cotton genome}

The availability of complete genome sequences from cotton provides an opportunity to identify and analyze the evolution and function of the CSLD proteins. $1923 \mathrm{Mb}$ (88.5\%), $1532 \mathrm{Mb}(90.4 \%)$ and $761.4 \mathrm{Mb}(99.95 \%)$ is anchored and oriented to 26 pseudochromosomes in G. 
hirsutum [45], to 13 pseudochromosomes in G. arboretum [48] and to 13 pseudochromosomes G. raimondii [20], respectively. Based on a homology-based protein search using confirmed functional CSLD proteins, we identified 23 full-length CSLD proteins from G. arboretum (six), G. hirsutum (11) and G. raimondii (six) (Table 1) and 86 CSLD proteins from 15 other plant species, as expected (Additional file 1: Table S1, Additional file 2). G. hirsutum has approximately twice as many CLSD proteins as G. arboreum or G. raimondii. In cotton, some CSLD proteins have one or two cellulose_synt (PF03552) domains and a zf-RING_4 (PF14570) domain, but the others have only one or two cellulose_synt (PF03552) domains (Fig. 1). The CSLD genes are distributed on six chromosomes (Dt_chr3, 5, 6, 8, 12 and At_chr8) and three scaffolds (S42.1, S2886.1, and S3941.1) in G. hirsutum, five chromosomes (Chr3, 4, 6, 8, and 11) in G. arboreum, and five chromosomes (Chr03, 04, 06, 08, and 12) in G. raimondii. Most chromosomes distributing the CSLD genes contain a single locus of CSLD genes, except chromosome 6 in G. arboreum, chromosome Dt_Chr8 in G. hirsutum and chromosome 08 in G. raimondii, which contain 2,3 and 2 CSLD gene loci, respectively (Fig. 2). The syntenic positions for G. arboreum and G. raimondii were compared with those of $G$. hirsutum (Fig. 2). One-to-two syntenic relationships were identified between G. arboreum or G. raimondii and G. hirsutum except for Chr4 (one-to-one syntenic relationship between G. arboreum and G. hirsutum), Chr06 (no syntenic relationship between G. raimondii and G. hirsutum) and Chr03 (one-to-three syntenic relationships between G. raimondii and G. hirsutum).

\section{Evolution of cotton CSLD proteins}

To reconstruct the phylogenetic trees, we used different alignment methods, evolutionary models, and multiple statistical-support measures (see the Methods section for details). Each alignment was analyzed with ProtTest 3.2 to select the most appropriate amino acid substitution model for inferred maximum likelihood (ML) phylogenetic trees. The LG + I + G + F model was chosen as the best model according to AIC, AICc and BIC criteria (Additional file 3: Table S2). To further verify the robustness of the phylogenetic trees reconstructed by PhyML, we inferred Bayesian

Table 1 Chromosomal locus ID and length of CSLD proteins in cotton

\begin{tabular}{|c|c|c|c|c|c|c|}
\hline Organism & CSLD protein & $G$ name $e^{a}$ & Exon & Locus ID & Strand & Length \\
\hline \multirow[t]{6}{*}{ G.arboreum } & Cotton_A_07355 & GaCSLD1 & 3 & Chr11:7526483-7529728 & + & 1006 \\
\hline & Cotton_A_05735 & GaCSLD2/3 & 2 & Chr3:41008,691-41012256 & - & 1160 \\
\hline & Cotton_A_02861 & GaCSLD2/3 & 3 & Chr8:99437456-99441339 & + & 1144 \\
\hline & Cotton_A_32285 & GaCSLD4 & 5 & Chr4:70165526-70169404 & - & 1144 \\
\hline & Cotton_A_07935 & GaCSLD6 & 3 & Chr6:50169413-50173209 & - & 1104 \\
\hline & Cotton_A_20715 & GaCSLD5 & 3 & Chr6:48564230-48568215 & + & 1175 \\
\hline \multirow[t]{11}{*}{ G.hirsutum } & CotAD_11457 & GhCSLD1 & 3 & Dt_Chr6:3724390-3727635 & - & 1006 \\
\hline & CotAD_67882 & GhCSLD1 & 3 & Scaffold3941.1:14327-17572 & - & 1006 \\
\hline & CotAD_04035 & GhCSLD2/3 & 2 & Dt_chr5:12149740-12153305 & + & 1143 \\
\hline & CotAD_56339 & GhCSLD2/3 & 2 & Scaffold2886.1:98206-101771 & + & 1143 \\
\hline & CotAD_31893 & GhCSLD2/3 & 3 & At_chr8:11464660-11468543 & + & 1144 \\
\hline & CotAD_24032 & GhCSLD2/3 & 3 & Dt_chr3:27558271-27562152 & + & 1144 \\
\hline & CotAD_28379 & GhCSLD4 & 4 & Dt_chr12:6056272-6060210 & - & 1121 \\
\hline & CotAD_17594 & GhCSLD6 & 3 & Dt_chr8:49809841:49813637 & + & 1104 \\
\hline & CotAD_16292 & GhCSLD5 & 3 & Dt_chr8:38197719-38201702 & - & 1175 \\
\hline & CotAD_41814 & GhCSLD6 & 3 & Dt_chr8:48706763-48710552 & - & 1104 \\
\hline & CotAD_11976 & GhCSLD5 & 3 & Scaffold42.1:2430018-2434003 & + & 1175 \\
\hline \multirow[t]{6}{*}{ G.raimondii } & Gorai.006G220600.1 & GrCSLD1 & 3 & Chr06:47301257-47304509 & + & 968 \\
\hline & Gorai.004G257300.1 & $\operatorname{GrCSLD} 2 / 3^{b}$ & 3 & Chr04:59350347-59355312 & + & 1143 \\
\hline & Gorai.003G052200.1 & $\operatorname{GrCSLD} 2 / 3^{\mathrm{b}}$ & 4 & Chr03:7953077-7958739 & - & 1144 \\
\hline & Gorai.012G137800.1 & GrCSLD4 & 4 & Chr12:31115815-31119908 & - & 1121 \\
\hline & Gorai.008G142900.1 & GrCSLD5 & 3 & Chr08:39398378-39402804 & - & 1174 \\
\hline & Gorai.008G223700.1 & GrCSLD6 & 4 & Chr08:51011238-51015956 & - & 1104 \\
\hline
\end{tabular}

${ }^{a}$ Gene names refer to the phylogenetic tree of CSLD proteins in Fig. 3

${ }^{\mathrm{b}}$ CSLD2 and CSLD3 are designated CSLD2/3 because of two closely related isoforms in $A$. thaliana 


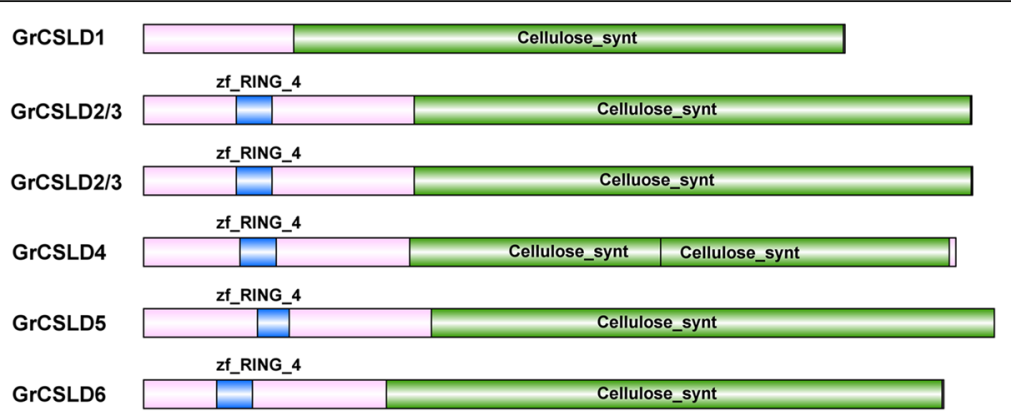

Fig. 1 The domain architecture illustrated using IBS software [112]. GrCSLD, CSLD protein in G. raimondii according to the phylogenetic tree of CSLD proteins in Fig. 3; zf-RING_4, zf-RING domain (blue); cellulose_synt, cellulose_synt domain (green). GrCSLD2/3, GrCSLD5 and GrCSLD6 have a cellulose_synt and a zf-RING_4 domain. GrCSLD1 only contains a cellulose_synt domain, and GrCSLD4 includes a zf-RING_4 and two cellulose_synt domains. Two GrCSLD2/3 s are shown in Fig. 1 because Gorai.004G257300.1, Gorai.003G052200.1, AT_CSLD2 and AT_CSLD3 form a monophyletic group (Fig. 3). The functional domains and positions of these domains were identified via sequence searches with the online programs SMART, Interpro and NCBI conserved domain databases

phylogenetic trees under a mixed model using MrBayes, which integrated over all available substitution models instead of specifying an amino acid substitution model [49]. In this approach, each of the multiple amino acid substitution models contributes to the result in proportion to its posterior probability. A comparison of phylogenetic trees obtained from ML and Bayesian methods using Ktreedist is shown in Additional file 4: Table S3. Based on the K-scores and symmetric differences (Robinson-Foulds distance), the ML and Bayesian trees based on elision and Muscle alignments exhibited nearly identical topology and branch lengths (K-score, 0.09 and 0.13; symmetric difference, 4 and 5). However, the ML and Bayesian trees based on two alignments (Kalign and Mafft) exhibited greater topological differences than the elision and Muscle alignments.

The Bayesian tree based on elision alignments divided the CSLD proteins into five strongly supported clades: CSLD1, CSLD2/3, CSLD4, CSLD5 and CSLD6, as observed in the model plant $A$. thaliana $[12,35]$. Support for the key nodes increased when we used the elision strategy, which concatenates the multiple alignments, and the mixed model method of MrBayes, suggesting that the most reliable alignment positions consistently support a phylogeny in which the CSLD proteins are classed into five clades (Fig. 3). As with the analysis of the whole CSLD phylogenetic tree, the cotton CSLD phylogeny was robustly

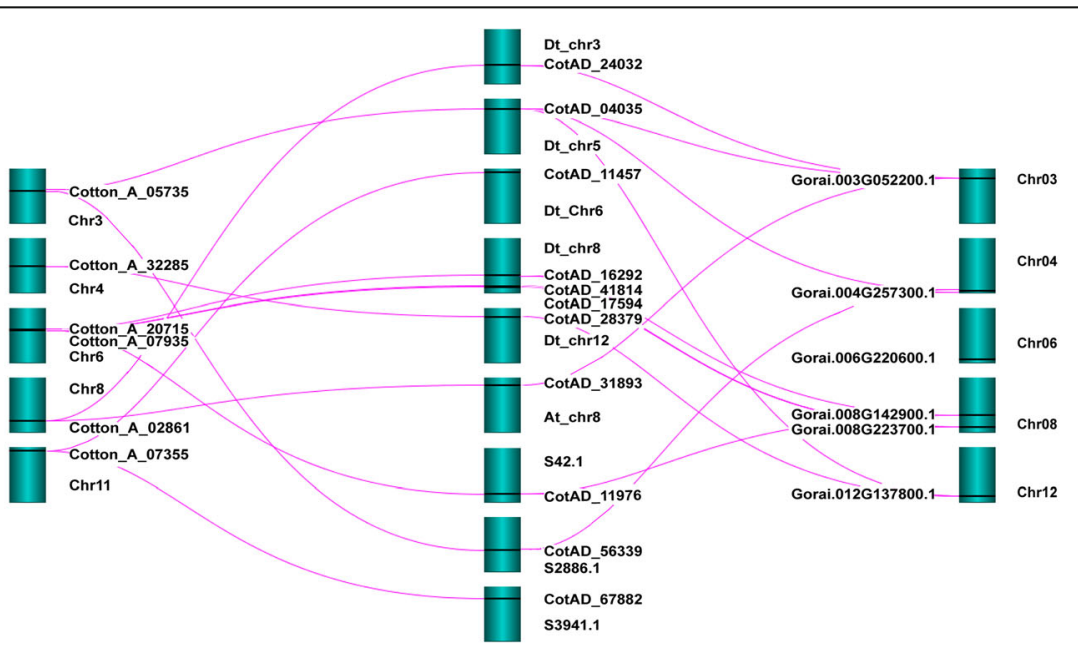

Fig. 2 Conserved syntenic positions in G. arboreum and G. raimondii compared with G. hirsutum using Strudel software. The pink lines show syntenic relationships between G. hirsutum and G. arboreum or G. raimondii. The positions of the CSLD genes on the respective chromosomes (dark cyan) are indicated using black lines 


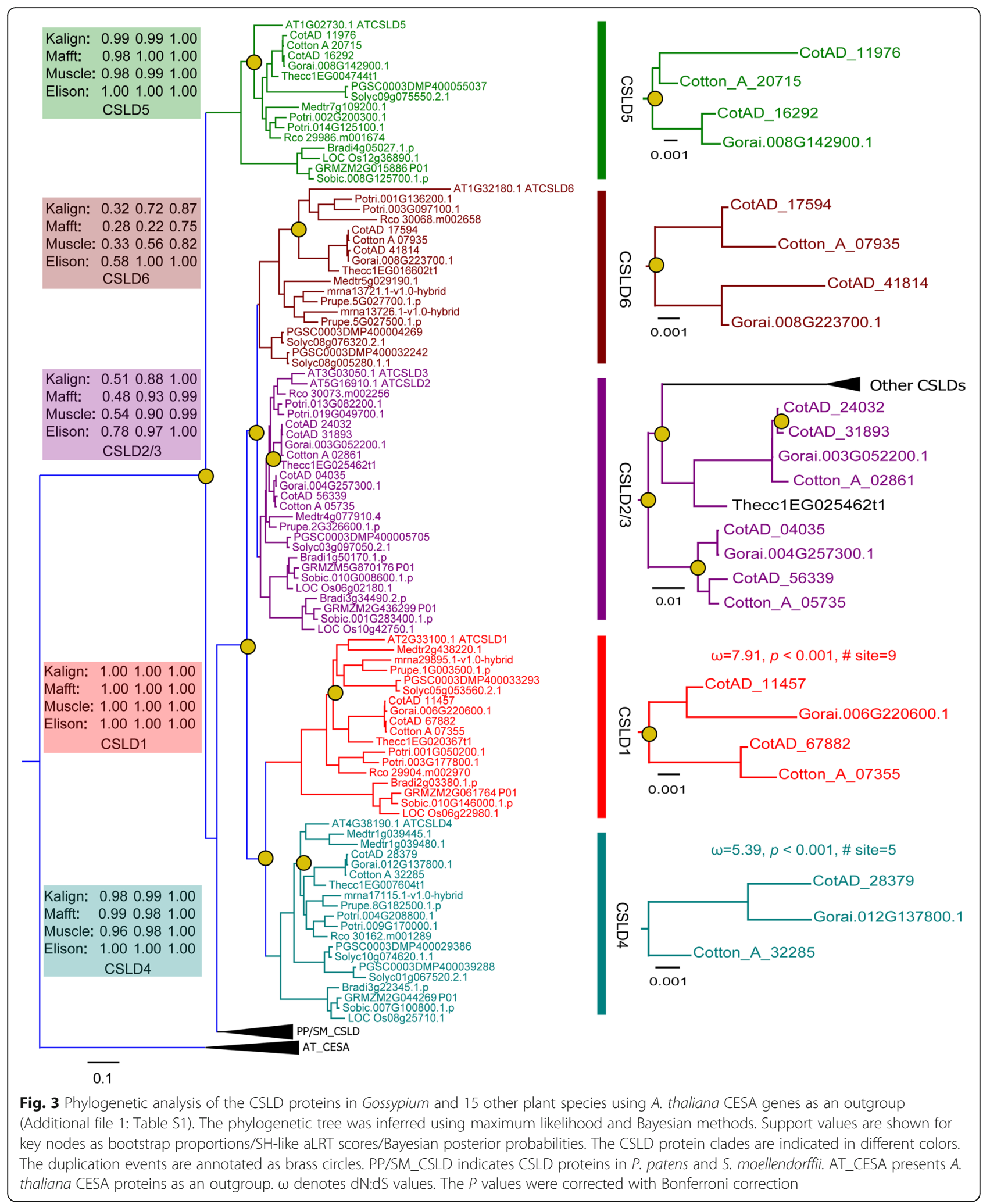

divided into five clades, and the support values of almost all nodes also increased when we used the elision strategy (Additional file 5: Figure S1). The topological differences based on the three alignments (Kalign, Mafft and Muscle) between the cotton CSLD trees inferred from ML and Bayesian methods are shown in Additional file 5: Figure S1. 
The topology of this phylogenetic tree shows that CSLD ancestral gene duplication occurred before the moss lineage diverged from vascular plants. Each of the two copies of the ancestral gene evolved separately, leading to the CSLD5 clade and the other CSLD clades (Fig. 3). The topology of the cotton CSLD tree is identical to that of the whole CSLD phylogenetic tree, which indicates that the cotton CSLD ancestor gene split into the CSLD5 clade and other CSLD clades, which were later divided into the CSLD1, CSLD2/3, CSLD4, and CSLD6 subclades via gene duplication. The CSLD proteins from the three Gossypium species form five monophyletic groups, each consisting of three or four CSLD proteins. CSLD1 and CSLD2/3 form sister groups to CSLD4 and CSLD6, respectively. The GhCSLD genes, except for CSLD4, duplicated once again recently (Fig. 3) through hybridization of the two ancestral species approximately 1.5 million years ago (MYA) [45]. Both $G$. raimondii and $G$. arboreum experienced an ancient hexaploidization event that is shared among the eudicots at 115-146 MYA and then underwent a cottonspecific whole genome duplication at 13-20 MYA [43, 48]. These conclusions support the presence of multiple CSLD gene copies in the three cotton species.

\section{Expression profiles of cotton CSLD genes}

Gene expression profiling can provide useful information for understanding gene function. To indicate whether CSLD genes have unique function among CESA/CSL superfamily, we performed the gene expression and qRTPCR analysis. The previous report has shown that OSCESA genes are highly expressed in most of the tissues examined, and OsCSL genes have the rather variable expression [13]. Based on the hierarchical clustering analysis, the CESA/CSL gene superfamily can be divided into five major groups in G. hirsutum, G. arboreum and G. raimondii (Figs. 4, 5 and 6). CSLD1 and CSLD4 exhibited high expression level in androecium \& gynoecium (A\&G) in the groups II of G. hirsutum. However, transcripts of CSLD1 and CSLD4 were zero or very small scores in other tissues of G. hirsutum, G. arboreum and G. raimondii (Figs. 4, 5 and 6, Additional file 6: Table S10, S11 and S12). CSLA2, CSLB, CSLG, CSLJ, CESA1/10, CESA3 and CESA4 were expressed in A\&G of G. hirsutum, but these genes, except CotAD_11650_GhCSLJ, also showed expression in other tissues (Fig. 4, Additional file 6: Table S10). This result indicated that expression of CSLD1 and CSLD4 appeared to have strong specificity, which was similar to the report that OSCSLD 3 and 5 (the orthologs of A. thaliana CSLD1 and CSLD4) showed strong expression in stamen (pollen) in rice and $A$. thaliana [13]. CSLD5 was primarily expressed at seedlings, root (radicle), stem, leaf and ovule, and other CSL genes were also expressed in these tissues (Figs. 4, 5 and 6, Additional file 6: Table S10, S11 and S12). GhCSLD6 expression was observed in all tissues, and had the low expression in old leaves, bract, ovule 30 dpa and ovule $40 \mathrm{dpa}$ (Fig. 4, Additional file 6: Table S10). CSLD 6 was expressed strongly in fiber, consistent with a previous report $[21,45]$. However, GrCSLD6 only exhibited strong expression at ovule (Fig. 6). GhCSLD2/3 genes fell into three distinct groups (in V), unlike GhCSLD1, 4 and 5, which were divided into one group (Fig. 4). CSLD2/ 3 showed primary expression in seed, seedlings, cotyledon, root, stem, leaf, corolla, ovule, fiber and boll shell (Figs. 4, 5 and 6, Additional file 6: Table S10, S11 and S12). CSLD2/3 genes in three distinct groups (two distinct groups in G. arboreum and G. raimondii) showed the different pattern of expression (Figs. 4, 5 and 6). These results implied that CSLD2/3 had multiple functions in synthesis of cell walls at the different development stages.

Generally, CESA genes showed an extensively high expression in all the tissues examined (Figs. 4, 5 and 6). Gh(a)CESA2/5/6/9, $1 / 10$ and 3 were expressed during primary cell wall biosynthesis at all tissues. Gh(a)CESA4, 7 and 8 were strongly expressed in secondary cell walls of tissues, for example, fiber $20 \mathrm{dpa}$. The expression patterns of CESA genes were similar to those of the OSCESA and ATCESA [13]. All of CSL genes showed relative tissue-specific expression, unlike CESA, which were expressed constitutively. The total expression of GhCSLA genes was highest in cotyledon $\mathrm{Y} 1$, and was followed by high expression in boll shell, with the lowest expression detected in the cotyledon Y2. In contrast, the total expression of GhCSLB genes was highest in cotyledon Y2. The total expression of $G h C S L C, D, E, G$ and $J$ genes was highest in stem Y1, A\&G (mostly contributed by CSLD1 and CSLD4), root, cotyledon $\mathrm{Y} 1$ and cotyledon $\mathrm{Y} 2$, respectively (Additional file 6: Table S10). The total expression of GaC$S L A$ and $E$ genes was highest in seedling. The total expression of $G a C S L B$ and $G$ genes was highest in seed. The total expression of $\mathrm{GaCSLC}$ was highest in seed $40 \mathrm{dpa}$. The total expression of GaCSLD (mostly contributed by CSLD5) was highest in leaf (Additional file 6: Table S11). GrCSLA genes showed an almost undetectable expression in all tissues. The total expression of $\operatorname{GrCSLB}$ was highest in seed $40 \mathrm{dpa}$. The total expression of GrCSLC and J was highest in mature leaf. The total expression of GrCSLD (mostly contributed by CSLD2/3) was highest in ovule 0 dap. The total expression of GrCSLE and $G$ was highest in seed (Additional file 6: Table S12). These results indicated that the expression of the CSL genes of the whole family often accumulated to high levels in one or more of the tissues for that the CSL members showed preferences, which were similar to a previous report in rice [13]. To gain more insights into whether the expression of CSLD genes is different, we performed qRT-PCR experiments with specific primers in G. hirsutum (Additional file 7: Table S13). Among all the 11 analyzed GhCSLD genes, one copy of GhCSLD2/3 (CotAD_24032) had the most prominent 


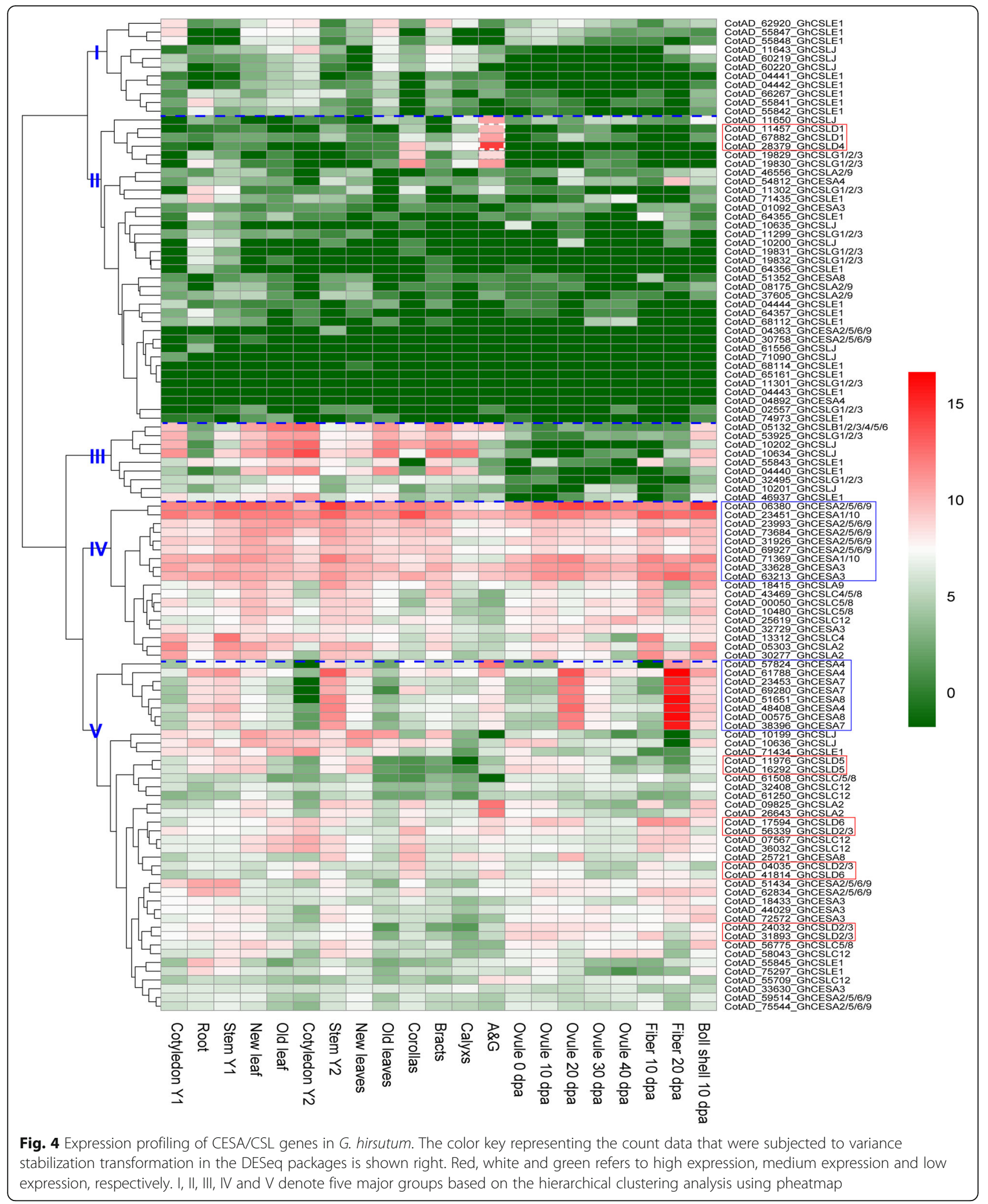

expression levels in all tissues (Fig. 7), followed by GhCSLD2/3 (CotAD_56339), GhCSLD2/3 (CotAD_31893) and GhCSLD5 (CotAD_16292). GhCSLD1 and GhCSLD4 showed an almost undetectable expression in all the tissues. GhCSLD6 was expressed at moderate levels. Overall, the results from the qRT-PCR expression data closely 


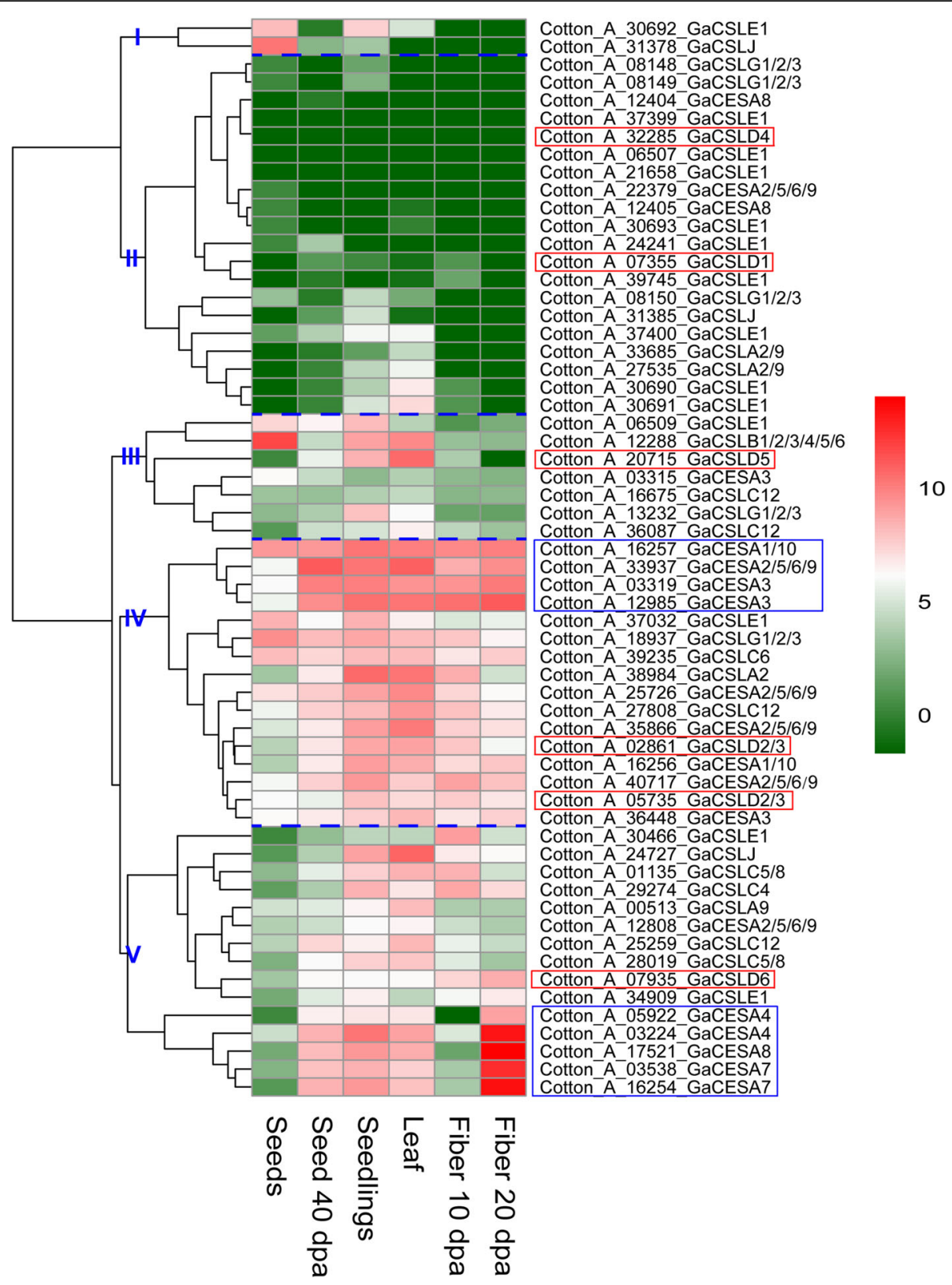

Fig. 5 Expression profiling of CESA/CSL gene in G. arboreum. The color key representing the count data that were subjected to variance stabilization transformation in the DESeq packages is shown right. Red, white and green refers to high expression, medium expression and low expression, respectively. I, II, III, IV and V denote five major groups based on the hierarchical clustering analysis using pheatmap

agreed with those of RNA-seq (Fig. 4). The results showed that CSLD genes exhibited the different pattern of expression compared with other CSL genes, and all the CSLD genes were differentially expressed in different cotton tissues under normal growth conditions, which indicated the functional diversification of CSLD genes in cotton.

\section{Identification of positive selection on the GrCSLD1 protein} Positive selection increases the frequency of mutations that confer a new fitness advantage to individuals carrying those mutations [50]. For protein-coding DNA sequences, positive selection is indicated by a ratio of nonsynonymous/synonymous mutation rates $(\omega=\mathrm{dN} / \mathrm{dS})$ greater than one [51]. Positive selection might occur if the gene is involved in plant-pathogen competition [52], if new and beneficial function emerged at the point of duplication [53], in response to stress [54], etc.

A site can be defined as undergoing long-term positive selection if it experiences positive pressure in most or all branches of the phylogeny $[54,55]$. We only focused on 


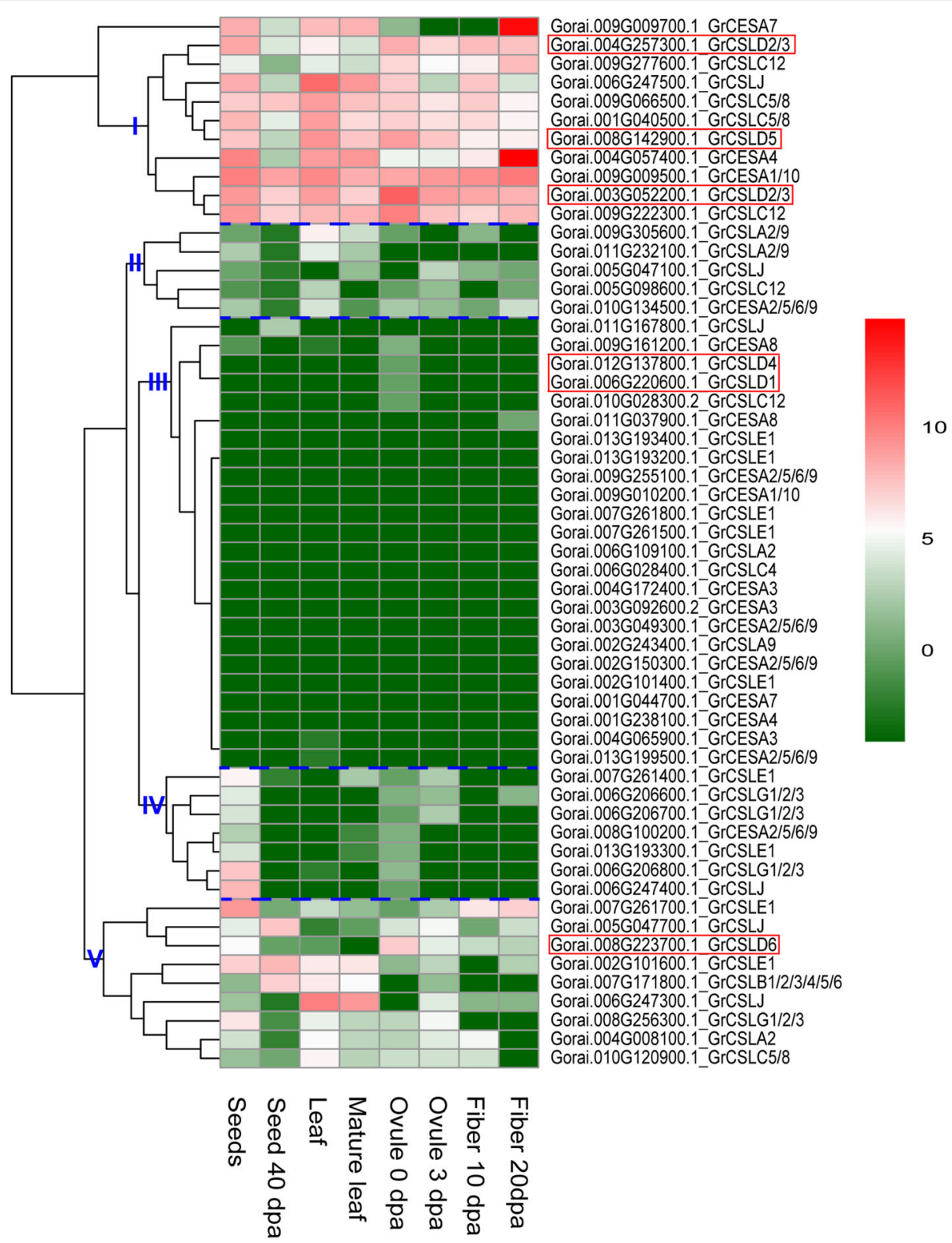

Fig. 6 Expression profiling of CESA/CSL gene in G. raimondii. The color key representing the count data that were subjected to variance stabilization transformation in the DESeq packages is shown right. Red, white and green refers to high expression, medium expression and low expression, respectively. I, II, III, IV and V denote five major groups based on the hierarchical clustering analysis using pheatmap

those branches defining the major clades of cotton, denoted CSLD1 to CSLD6 (Fig. 3). We used the Notung method [56] to infer gene duplication and determined long-term positive selection after duplication by applying the branch-site model at the clade level (across all branches in each specified clade). Two branches (CSLD1 and CSLD4) exhibited episodic positive selection after Bonferroni correction. Nine and five sites were identified as undergoing positive selection after duplication by the Bayes empirical Bayes analyses, respectively (Table 2). CSLD1 and CSLD4 also showed a long-term shift in positive selection across every branch of the cotton lineage.
Thirteen and two sites with significant evidence for positive selection were detected in the CSLD1 and CSLD4 clades, respectively. Amino acid residues of positive sites are shown in Table 2.

The positively selected sites in Table 2 were located on the predicted tertiary structure of the GrCSLD1 protein. K116 is spatially close to the class-specific region (CSR), and A244 is located on the $\beta 1$-strand (Additional file 8: Figure S3 and Additional file 9: Figure S4). C157, C795, Q894, A912, C917 and C947 are positioned in the transmembrane helices (TMHs). Furthermore, C795, A912 and C917 are located within transmembrane pore predicted by 


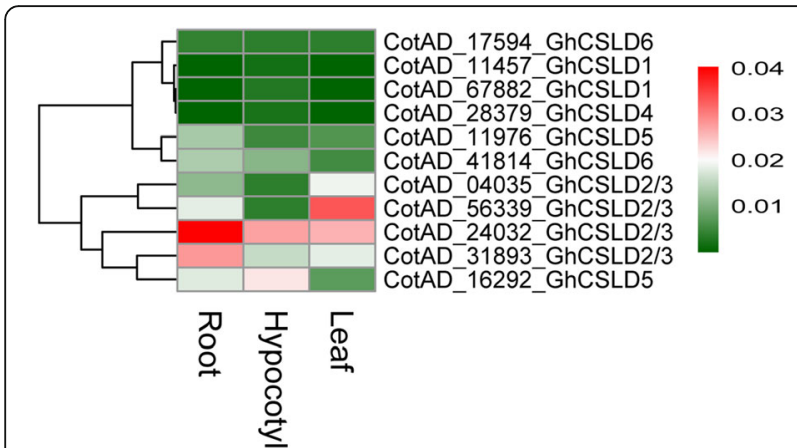

Fig. 7 Heat map of qRT-PCR in G. hirsutum. The color key representing relative expression level of CSLD genes by comparative $2^{-\Delta \Delta C T}$ method is shown right. Red, white and green refers to high expression, medium expression and low expression, respectively

MEMSAT (Additional file 8: Figure S3 and Additional file 9: Figure S4).

\section{Structure of GrCSLD1 protein}

In A. thaliana, CSLD1 and CSLD4 are both expressed at high levels in the pollen tubes and mature pollen grains, and the synthesis of the pollen tube wall is significantly reduced in CSLD1 and CSLD4 mutants [30]. The CSLD1 and CSLD4 proteins are localized in the Golgi apparatus before germination and are then transported to the plasma membrane at the pollen tube tip [30,34]. These results suggest that the CSLD1 and CSLD4 proteins probably present distinct cellulose synthesis activities at the apical plasma membrane during tip growth in pollen tube cells. To gain insights into the function of CSLD1 proteins in cotton, a structural model was built via template-based and template-free modeling.

I-TASSER, Phyre2 and Robetta (prediction of domains with comparative modeling, see Methods) were all used with BcsA [57] as the primary template. The model validation scores of the full-length GrCSLD1 protein are shown in Additional file 10: Table S15. We identified the top-scoring model predicted by Robetta. Structural alignment of the top-scoring model with BcsA gave a TM-score of 0.65 , suggesting that GrCSLD1 and BcsA share the same fold [58].

All known CSLD proteins are classified as GT2 family in the CAZy database [9]. GT2 proteins are predicted to be inverting enzymes, that is, the configuration of the anomeric sugar carbon is inverted during the transfer reaction [59]. The GT2 family includes cellulose synthase, $\beta-1,4-$ mannan synthase, and chitin synthase. The GT domain has a GT-A fold consisting of seven $\alpha$-helices, three amphipathic interface (IF) helices (IF1-3) attached to the transmembrane region, and a seven-stranded $\beta$ sheet that resembles a Rossmann fold [57, 60]. However, a three-dimensional structure of a cotton CSLD protein has not been solved.

Our results show that the predicted GrCSLD1 structure contains $31 \alpha$-helices and $9 \beta$-strands (Figs. 8 and 9 , Additional file 9: Figure S4). The GrCSLD1 core domain was superimposed with GhCESA1 [61] and BcsA [57] using MatchMaker in UCSF-Chimera [62]. In the superimposition of GrCSLD1 with BcsA and GhCESA1, structure matching of the domain included 8 helices $(\alpha 2, \alpha 4$, $\alpha 6, \alpha 7, \alpha 8, \alpha 12, \alpha 13, \alpha 17)$ and $7 \beta$-strands that form the $\beta$-sheet ( $\beta 1, \beta 2, \beta 3, \beta 4, \beta 5, \beta 6, \beta 7)$, with an overall root mean square deviation (RMSD) of $1.84 \AA$, and 6 helices $(\alpha 2, \alpha 6, \alpha 7, \alpha 8, \alpha 12, \alpha 13)$ and $6 \beta$-strands that form the $\beta$-sheet $(\beta 1, \beta 2, \beta 3, \beta 4, \beta 5, \beta 6)$ with an overall RMSD of 2.74 $\AA$, respectively (Figs. 9 and 10, Additional file 9: Fig. S4). Therefore, the core domain of GrCSLD1 contains 8 $\alpha$-helices $(\alpha 2, \alpha 4, \alpha 6, \alpha 7, \alpha 8, \alpha 12, \alpha 13, \alpha 17)$ and the seven-stranded $\beta$-sheet that forms a Rossmann fold (Fig. 9, Additional file 9: Figure S4). The core domains of GrCSLD1 and GhCESA1 show structural congruence, even though the GhCESA1 structure was not used for prediction of the GrCSLD1 model. By analogy to GhCESA1, the catalytic pocket of GrCSLD1 comprises the closely arranged, conserved DD, DCD, TED and QVLRW motifs. The $\alpha 13$ helix is positioned near these conserved motifs, corresponding to IF2, which interacts with the cellulose acceptor substrate (Fig. 10a) in BcsA [57]. GrCSLD1 only contains a cellulose_synt domain (Fig. 1), and the CSLD2/3 genes also have been suggested to be involved in mannan synthesis during cotton fiber cell development [47]. These results suggest that GrCSLD1 belongs to GT2 and probably participates in the biosynthesis of cellulose, mannan or other polysaccharides.

\section{Discussion}

The CSLD proteins, which feature a conserved D, D, D, QXXRW motif, belong to the ancient cellulose synthase superfamily $[10,12]$. In addition to CESA, the CSLD

Table 2 Amino acid sites using the branch-site model under positive selection

\begin{tabular}{|c|c|c|c|}
\hline Foreground & $\omega$ & $P$-value & Site under positve selection \\
\hline CSLD1 branch & 7.91 & $P<0.001$ & S29*, K116*, C157*, A244*, C795*, Q894*, A912* , C917*, C947* \\
\hline CSLD1 clade & 3.56 & $P<0.001$ & D2**, N6*, S8* S29*, K116*, C157*, N188*, A244* C795*, Q894*, A912*, C917*, W952* \\
\hline CSLD4 branch & 5.39 & $P<0.001$ & T314*, F929*, Q953*, G1046*, G1104* \\
\hline CSLD4 clade & 2.57 & $P<0.001$ & Q953*, G1104* \\
\hline
\end{tabular}

Sites are numbered according to the full GrCSLD1 coding sequence. Sites with posterior probabilities greater than $0.95\left(^{*}\right)$ and $0.99\left(^{* *}\right)$ are shown 

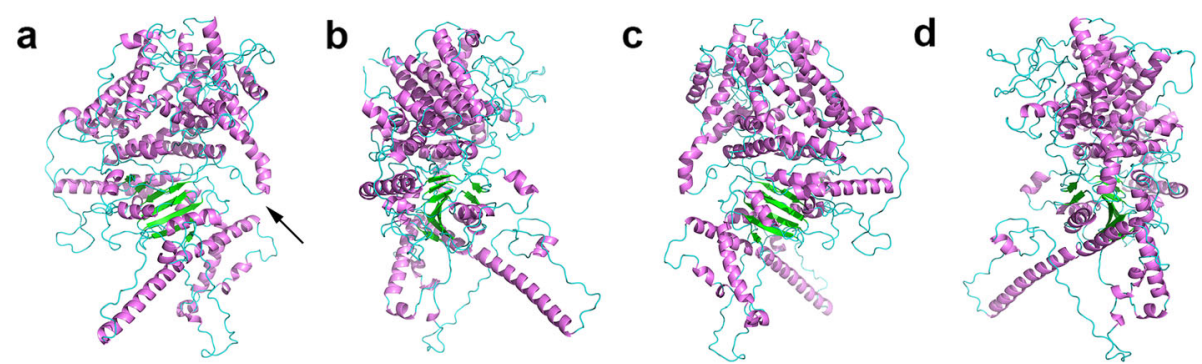

Fig. 8 Structural model of GrCSLD1. The indicated structures are progressively rotated $90^{\circ}$ from left to right (a-d). The black arrow indicates the active site motifs in (a)

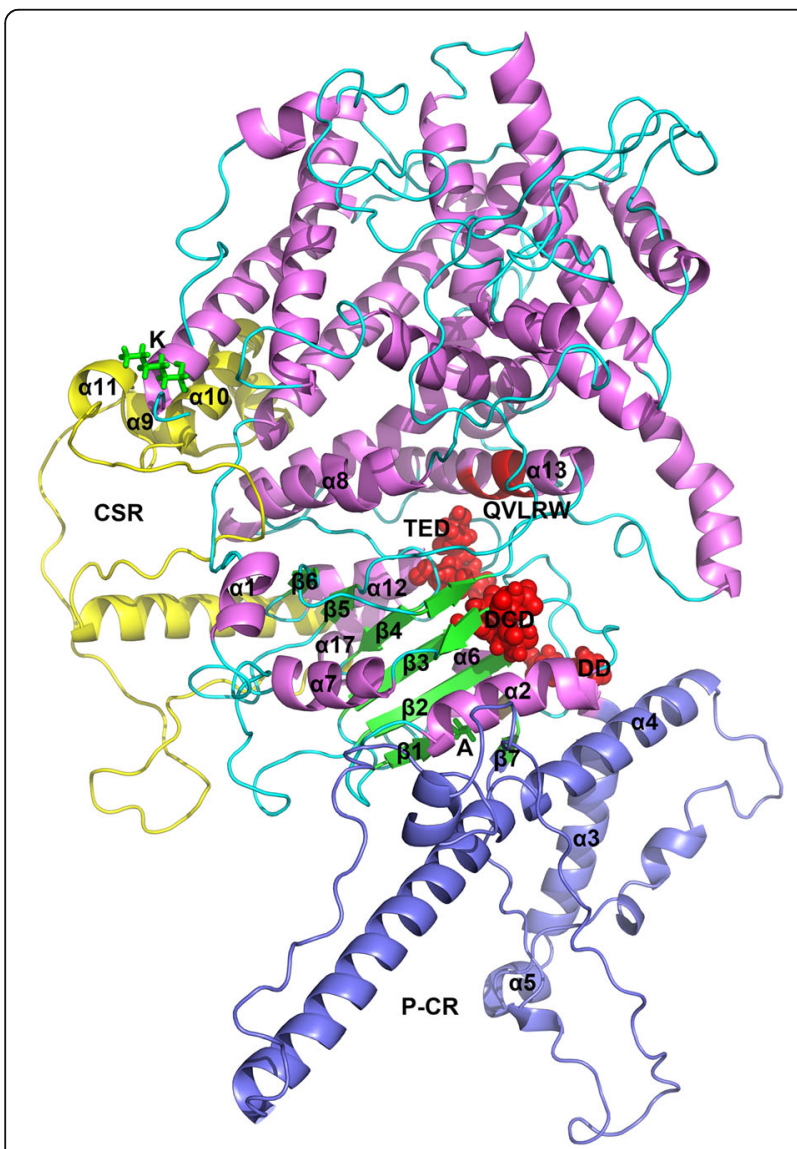

Fig. 9 Structural model of GrCSLD1 showing the positions of the amino acid residues under positive selection, plant-specific regions and active site motif. The structures of GrCSLD1, P-CR and CSR are colored violet, light blue and yellow, respectively. The core domain contains 8 a-helices (a2, a4, a6, a7, a8, a12, a13, and a17) and the seven-stranded $\beta$-sheet. The numbering of the a-helices and $\beta$-strands is based on their order in the secondary structure of GhCESA1 (Additional file 9: Fig. S4). Red highlights DD, DCD, TED (spheres) and QVLRW. The sites ( $K$ and $A$ ) under positive selection are shown as green sticks proteins are the only members of the superfamily with a zf-RING (Fig. 1) domain in the N-terminal region, which is thought to function in protein-protein interactions [12]. The CSLD proteins remain poorly understood despite their importance for tip-growing cells and stem growth.

\section{Conserved synteny of CSLD genes distributed across the cotton genome}

We identified 23 full-length CSLD proteins: six, six and 11 from G. arboreum, G. hirsutum and G. raimondii, respectively (Table 1 ). The CSLD genes are distributed across several chromosomes. Conserved synteny of all CSLD genes was observed between G. arboreum and G. hirsutum. These are one-to-two syntenic relationships, except for CSLD4 genes on Chr4, which had a one-toone syntenic relationship between G. arboreum and G. hirsutum (Fig. 2). The one-to-one syntenic relationship of CSLD4 genes exists because CSLD4 is a single-copy gene in G. hirsutum. However, conserved synteny of CSLD1 genes was not detected between G. raimondii and G. hirsutum (Fig. 2). There are one-to-two syntenic relationships for CSLD4, CSLD5, CSLD6 and one copy of $C S L D 2 / 3$. No syntenic relationships were identified for $C S L D 1$, perhaps because the synteny hits are concealed by the annotation string search in SyMAP [63]. The one-to-three syntenic relationships in one copy of CSLD2/3 on Chr03 between G. raimondii and G. hirsutum might be caused by two closely related isoforms (ATCSLD2/3) in A. thaliana. One-to-two syntenic relationships were mostly identified between G. arboreum or G. raimondii and G. hirsutum because the G. hirsutum genome is derived from hybridization of $\mathrm{A}_{2}$ and $\mathrm{D}_{5}$ genome ancestors [45]. Comparison of the synteny map and CSLD phylogeny showed that most CSLD genes in synteny blocks form a monophyletic clade, indicating that CSLD genes have been conserved over considerable time, whereas genes within the clades have evolved.

Recent studies have shown that the present allotetraploid G. hirsutum was derived from hybridization of $\mathrm{A}_{2}$ and $\mathrm{D}_{5}$ genome ancestors approximately 1.5 MYA [45]. 

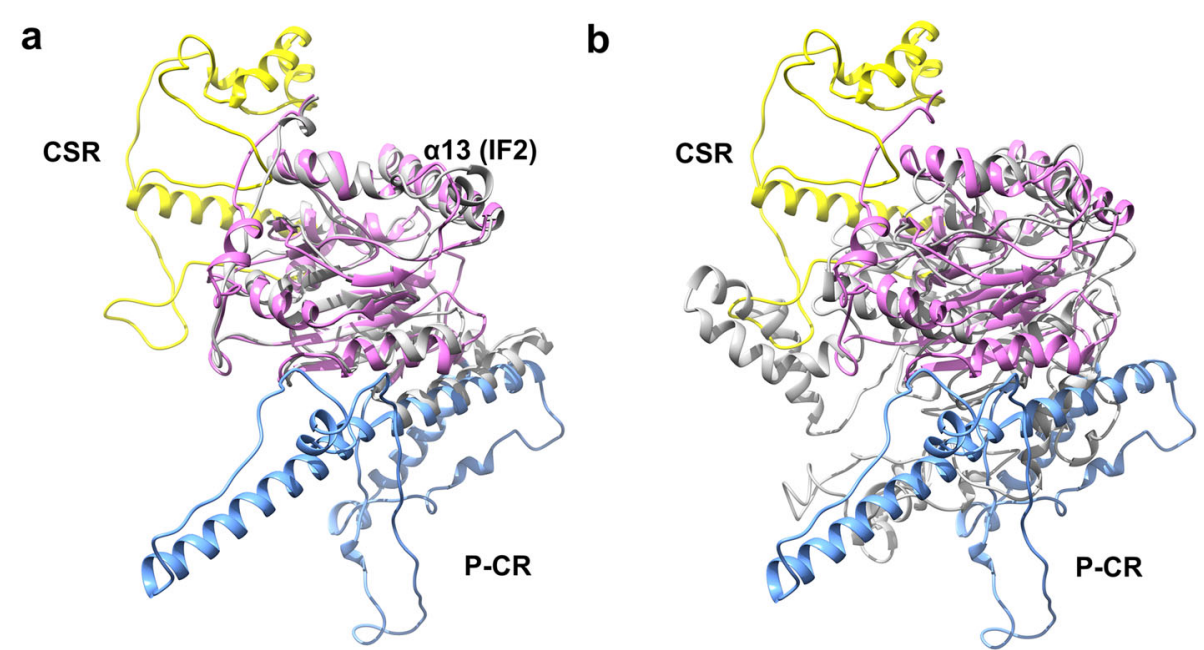

Fig. 10 Superimposition of the core domain of GrCSLD1 with BCSA and GhCESA1. Residues 171 to 727 of GrCSLD1 were superimposed with residues 119 to 394 of BCSA (a) and residues 220 to 725 of GhCESA1 (b) using MatchMaker in UCSF-Chimera. All proteins adopt a GT-A fold. a The overall RSMD value between matched $C_{a}$ atoms was $1.84 \AA$, and a13 denotes the motif corresponding to IF2 of BCSA. $\mathbf{b}$ The overall RSMD value between matched $C_{a}$ atoms was $2.74 \AA$. GrCSLD1, BCSA and GhCESA are colored violet, gray and gray, respectively. P-CR and CSR are indicated in light blue and yellow

The G. arboreum and G. raimondii genomes have undergone two rounds of whole-genome duplication (WGD), which are estimated to have occurred approximately 1320 and 115-146 MYA, respectively [43, 48]. The ancient duplication event corresponds to the ancient hexaploidization event shared among eudicots [64]. G. arboreum and G. raimondii have approximately the same number of CSLDs as A. thaliana, Oryza sativa and Zea mays, and G. hirsutum has approximately twice as many as G. arboreum or G. raimondii. Moreover, the CSLD proteins of cotton and those of Physcomitrella patens and Selaginella moellendorffii form a sister group to CSLD5. These results also suggest that CSLD genes are conserved in cotton and an ancient gene family, and the expansion of CSLD genes is associated with WGD.

\section{Reconstructing phylogenetic trees of CSLD proteins}

The quality of the multiple sequence alignment (MSA) has a profound impact on the robustness of a given phylogenetic tree [65]. Because genes evolve at different rates, some regions of an alignment are very well conserved and suitable for phylogenetic analysis, whereas others are full of gaps and very divergent. These divergent regions cannot be precisely aligned and thus must be removed prior to phylogenetic analysis [66]. Phylogenetic reconstruction produces an estimate of the true history by examining alternative trees and then quantifying the extent to which sequence data support or reject different phylogenetic results. Maximum likelihood [67] and Bayesian inference [68] are the most popular methods to build phylogenetic trees. Therefore, we used multiple alignment strategies (Kalign, Mafft and Muscle), support measures (SH-like approximate likelihood ratio tests, nonparametric bootstrap proportions and Bayesian posterior probabilities) and alignment trimming (Gblocks) in the current study.

Our results suggest that the cotton CSLD phylogenetic trees inferred from ML and Bayesian, based on three alignments and an elision strategy can be divided into five strongly supported clades. The division of the phylogenetic tree of the CSLD proteins into five clades is also robust with respect to other factors that are known to affect phylogenetic tree accuracy, including statisticalsupport measures and evolutionary models. However, the topology of the cotton CSLD5 and CSLD2/3 trees inferred from ML and Bayesian, based on two alignments (Kalign, Mafft) and the elision strategy, had some differences (Additional file 5: Figure S1). The Bayesian trees of CSLD5 and CSLD2/3 trees based on two alignments (Kalign and Mafft) have polytomies. We consider these soft polytomies because the trees from other methods were fully binary. The appearance of polytomies may be due to contradictory results from conflicting data and a lack of information regarding the true bifurcating pattern of the proteins [69]. The ML and Bayesian trees based on Muscle and elision alignments showed almost identical topology and best estimate the true evolution of CSLD proteins. We used the ML tree based on Muscle (bootstrap branch supports) to infer duplication and evaluate positive selection. Cotton CSL genes are involved in the synthesis of cell wall matrix polysaccharides surrounding cellulose microfibrils in cotton [20]. CSLD2/3 and CSLD 6 but not CSLD1 and CSLD4 genes are expressed strongly during fiber development $[21,45]$. The 
CSLD2/3 proteins also have been suggested to be involved in mannan synthesis during cotton fiber cell development [47]. These results imply that CSLD proteins may participate in the biosynthesis of cellulose, mannan or other polysaccharides.

\section{Characterization of CSLD gene family}

A. thaliana and rice provide a reference point for understanding the function of cotton CSLD proteins. CSLD proteins in $A$. thaliana might be involved in cellulose synthesis in tip-growing cells (pollen tubes and root hairs), stem growth and mannan synthesis, which suggests that CSLD proteins have acquired different functions.

To demonstrate the functional characterization of CSLD genes, we performed the gene expression and qRT-PCR analysis. The previous report has shown that OSCESA genes are highly expressed, and OsCSL genes have the rather variable expression [13]. Almost all CESA genes in cotton exhibited high expression in all tissues examined, implying that their major roles in the biosynthesis of cellulose, the core structural component of the cell wall. CESAs (2/5/6/9, 1/10 and 3) and CESAs (4, 7 and 8) were strongly co-expressed (in IV and V group), suggesting that CESAs $(2 / 5 / 6 / 9,1 / 10$ and 3$)$ and CESA (4, 7 and 8) may form two synthesis complexes involved in primary and secondary cell wall synthesis, as observed in the model plant $A$. thaliana $[2,12]$ and rice [13]. The results also were consistent with the report that CESA1, 2, 7, 8 (the orthologs of $A$. thaliana CESA8, 4, 7 and 7, respectively) are associated in the cellulose biosynthesis secondary cell wall, whereas CESA3, 5, 6, 9 and 10 (the orthologs of $A$. thaliana CESA3, 2/5/6/9, 1/ $10,2 / 5 / 6 / 9$ and 3 , respectively) participate in primary cell wall synthesis in cotton [19-22].

One copy of GrCESA1/10 gene exhibited high expression in all tissues, and one copy of GrCESA4 and 7 was strongly expressed in fiber 20dpa, which suggesting that there are fewer GrCESA proteins involved in the biosynthesis of cell wall (Fig. 6, Additional file 6: Table S12). Compared with CESA genes, CSLD genes were expressed in one or more of the tissues.

In CSL gene superfamily, The total expression of CSLD genes was different from other $C S L$ genes. Furthermore, different copy of $C S L$ genes showed different expression patterns. CSLD1 and CSLD4 were strongly co-expressed in A\&G, and showed tissue-specific expression (Figs. 4 and 7), suggesting that CSLD1 and CSLD4 may form a synthesis complex involved in polysaccharides. The overall expression of CSLD2/3 and CSLD5 genes was highest in root and leaf, respectively (Additional file 6: Table S10). These results were consistent with the previous reports in A. thaliana and rice [13, 30, 34]. CSLD 6 was expressed strongly in fiber, consistent with a previous report [21, 45]. GrCSLD6 only exhibited expression at ovule (Fig. 6); however, ATCSLD6 appears to be a pseudogene [31]. These results imply the CSLD genes show relative tissue-specific expression, indicating their potentially different function in the biosynthesis of polysaccharides.

\section{Spatial distribution of amino acids under positive selection in GrCSLD1}

Branch-site model analyses showed differences in the selection pressure on major clades, which implies that some sites in CSLD proteins from cotton are subject to different constraints during the evolutionary process. These constraints are imposed by the varied functional roles and evolutionary origins of CSLD proteins. CSLD2/3, CSLD5 and CSLD6 were found to have undergone relaxed purifying selection. However, CSLD1 and CSLD4 showed episodic positive selection and long-term shift positive selection across every branch of the cotton lineage after gene duplication. CSLD1 (ATCSLD4), CSLD2 and CSLD4 (ATCSLD5) showed a strong positive selection signal in grasses [54]. It is possible that there are different evolutionary pressures in cotton and grass. The CSLD1 and CSLD4 genes are required for normal growth of pollen tubes in A. thaliana, possibly by participating in pollen tube cellulose synthesis [30, 34]. The gene expression and qRT-PCR analysis showed that CSLD1 and CSLD4 genes only exhibited strongly expression in A\&G, and have the different expression patterns from other CSLD genes, which imply that CSLD1 and CSLD4 have the potentially different function in the biosynthesis of polysaccharides, compared with other CSLD proteins. Our results suggest that CSLD1 and CSLD4 genes probably evolved new functions after gene duplication through long-term shifts in positive selection.

The recently reported three-dimensional structure of the A and B subunits of a bacterial cellulose synthase complex from Rhodobacter sphaeroides [57] and a computational model of cotton GhCESA1 [61] provide an opportunity to define the three-dimensional distribution of the positively selected sites in GrCSLD1. However, the distinct functions of CSLD proteins remain unknown. Some reports have shown that CSLD proteins are associated with cellulose and mannan biosynthesis [29-31, 47]. There is no direct evidence that the GrCSLD1 protein has a distinct catalytic function. Functional characterization based on the predicted three-dimensional structure of GrCSLD1 proteins is extremely difficult. GrCSLD1 contains a conserved cellulose_synt domain (Fig. 1) and exhibits a phylogenetic relationship with other functionally known CSLD proteins in other plants and structural similarity with BcsA and GhCESA1. The three-dimensional structure of GrCSLD1 is predicted to contain a Rossmann fold and has a conserved D, D, D, QXXRW motif (Fig. 9). These results imply that GrCSLD1 belongs to GT2; 
however, the definite role of GrCSLD1 is not known. We only suggest GrCSLD1 probably participates in the biosynthesis of cellulose, mannan or other polysaccharides.

The amino acid residues identified as under positive selection in the CSLD1 lineage are located on a region adjacent to the CSR, $\beta 1$-strand and TMHs in the structure of GrCSLD1 (Fig. 9, Additional file 8: Figure S3 and Additional file 9: Figure S4). The structure of GrCSLD1 revealed that CSR and $\mathrm{P}-\mathrm{CR}$ fold into distinct subdomains within the cytosolic region. The CSR region probably helps stabilize CESA assembly through non-covalent interactions [61]. K116, a residue under positive selection that is spatially adjacent to the CSR region, may help stabilize CSLD1 assembly into complexes with other CSLDs (CSLD1 and CSLD4 genes are strongly expressed in $A \& G)$, similar to CESAs. Interestingly, A244 is positioned on a $\beta 1$-strand within the core domain of GrCSLD1, which suggests that this residue has the potential to influence GrCSLD1 activity. C157, C795, Q894, A912, C917 and $\mathrm{C} 947$ are positioned in the predicted TMHs and within the transmembrane pore that is involved in the extrusion of the nascent polysaccharide across the cell membrane (Additional file 8: Figure S3 and Additional file 9: Figure S4). Our analyses suggest that the residues of GrCSLD1 under positive pressure have relatively significant influence on enzyme activity or function, and on the fine structure of the polysaccharide that enzyme synthesizes. The specific roles of these sites under positive selection in GrCSLD1 remain unknown and warrant further research.

Recent reports show that CSLD proteins are not included in the modules of cell wall polymer biosynthesis in rice [70] and that the CSLD proteins are not interacted with cellulose synthase complexes in cotton [71]. CSLD1 and CSLD4 genes may be specifically involved in biosynthesis of cellulose at the tip of growing pollen tube and are highly expressed in mature pollen grains and pollen tubes in A. thaliana [30]. These results also suggest that CSLD1 and CSLD4 probably function as a complex in cellulose biosynthesis.

\section{Conclusions}

The CSLD family remains relatively uncharacterized within the community, and many questions about its evolutionary history and function remain. In this study, we performed rigorous phylogenetic analyses with maximum likelihood and Bayesian methods to resolve the phylogenetic topology of CSLD proteins in cotton. Tests for positive selection, gene expression profiling and qRT-PCR analysis were performed in the context of determining characterization of CSLD genes, compared with CESA and other CSL genes. These analyses were supplemented with GrCSLD1 homology modeling to provide a structural context for the evolutionary and functional characterization of CSLD proteins. These data provide a basis for understanding the evolutionary history and 3D modeling of CSLD proteins in cotton.

\section{Methods \\ Identification of CSLD proteins}

We used confirmed functional protein sequences of CSLD in A. thaliana as queries to identify new CSLD protein homologs from fully sequenced genomes of cotton (G. arboreum, G. hirsutum and G. raimondii) and 15 other plant species (Additional file 1: Table S1) using BLASTP (E-value $\leq 1 \mathrm{E}-5$ ) [72]. The G. arboreum and G. hirsutum sequences were retrieved from CGP (http:// cgp.genomics.org.cn). G. raimondii and 15 other fully sequenced plant genomes were retrieved from Phytozome (V11) [73]. To distinguish CSLD from CESA and other CSLs, we used the hit candidates of CSLD to search against the proteome of $A$. thaliana from Phytozome using BLASTP. Each true CSLD is expected to identify a CSLD from $A$. thaliana as the top hit according to the nomenclature of the cellulose synthase superfamily of $A$. thaliana [12]. CESA has more exons than CSLD. The conserved domains of all obtained sequences were verified via sequence searches with the online program SMART [74], Interpro [75] and the NCBI conserved domain databases [76]. Synteny blocks between G. hirsutum and G. arboreum or G. raimondii were detected using SyMAP by default [63] and visualized with Strudel [77].

\section{Multiple sequence alignment}

The CSLD protein sequences were aligned using Kalign v2.04 with default parameters [78], E-INS-I methods from Mafft v7.215 [79], and Muscle v3.8.31 [80]. Divergent and ambiguously aligned regions from the resulting alignments were trimmed with Gblocks v0.91b [81] prior to phylogenetic analysis. We also produced an elision alignment by concatenating all three individual Gblocksprocessed alignments [82].

\section{Phylogenetic analysis}

Maximum likelihood phylogenetic trees were reconstructed using PhyML v3.0 [83], with the best-fit models of amino acid substitution selected by ProtTest v3.2 [84]. Branch supports were estimated using SH-like approximate likelihood ratio tests [85] and non-parametric bootstrap proportions (500 replicates). Bayesian phylogenies were reconstructed using MrBayes v3.2.5 [49]. We integrated out amino acid substitution models (prset aamodelpr $=$ mixed) and assumed a model of discretegamma distributed rate variation across sites. The Markov chain was sampled every 100th generation, and the initial $25 \%$ of samples were discarded as burn-in, with the remaining samples used to generate the consensus tree. We assessed chain convergence by running two simultaneous, independent analyses and terminated the analysis 
when the average standard deviation of split frequencies between the two runs fell below 0.01. Phylogenetic trees obtained from ML and Bayesian reconstructions were compared regarding both topology and branch lengths using Ktreedist [86].

\section{Gene expression and qRT-PCR analysis}

The high-throughput RNA-sequencing data were downloaded from Short Read Archive of the National Center for Biotechnology Information (http://www.ncbi.nlm.nih.gov/ sra, Additional file 11: Table S4, S5 and S6). The RNA-seq reads were mapped to the reference cotton genome with TopHat2 [87]. The set of files containing mapped reads from TopHat2 were sorted and indexed using samtools [88]. The overlap of reads with genes were counted using HTseq-count [89]. The counts of genes were estimated normalization and dispersions, and were transformed to variance stabilization data with DESeq (Additional file 12: Table S7, S8, and S9) [90, 91]. We produced the heatmaps based on the variance stabilization transformed data for CESA/CSL gene superfamily of cotton using pheatmap package (pheatmap: Pretty Heatmaps, $R$ package version 1.0.8, https://CRAN.R-project.org/package=pheatmap). We used PF03552 (Cellulose_synt) and PF00535 (Glycos_transf_2) as queries to identify new CESA/CSL protein homologs from fully sequenced genomes of cotton (G. arboreum, G. hirsutum and G. raimondii) using HMMER $3.1 \mathrm{~b} 2$ package [92]. The CESA/CSL protein sequences were aligned using Muscle v3.8.31 [80]. Divergent and ambiguously aligned regions from the resulting alignments were trimmed with Gblocks v0.91b [81] prior to phylogenetic analysis. Maximum likelihood phylogenetic tree was reconstructed using PhyML v3.0 [83], with the best-fit model (JTT $+G+F)$ selected by ProtTest v3.2 [84]. The CESA/CSL protein sequences of A. thaliana were downloaded from the TAIR 10 database (https://www.arabidopsis.org) [93]. All the identified CESA/CSL genes in cotton (G. arboreum, G. hirsutum and $G$. raimondii) were provided specific names based on the orthologous sequence with $A$. thaliana (Additional file 13: Figure S2). G. hirsutum (Chinese cotton cultivar Yinshan 2, Henan Qiule Seed Industry Science\&Technology LTD., COM) were grown in a growth chamber at $28{ }^{\circ} \mathrm{C}$ with a $14 \mathrm{~h}$ light and $10 \mathrm{~h}$ dark cycle. When three fully expanded leaves appeared, root, hypocotyl and leaf were collected separately, frozen immediately in liquid nitrogen and stored at $-80{ }^{\circ} \mathrm{C}$ until RNA extraction. Each sample was preformed in three biological replicates. Total RNA was extracted from root, hypocotyl and leaf using Trizol reagent according to the manufacturer's instructions (TaKaRa), and treated extensively with RNase-free DNase I. The cDNA was synthesized from $1 \mu \mathrm{g}$ of total RNA using a First Strand cDNA Synthesis Kit (Invitrogen). The primers of CSLD genes from G. hirsutum designed for the qRT-PCR analysis are listed in Additional file 7: Table S13. QRT-PCR was performed as previously described [94,95]. The comparative $2^{-\Delta \Delta C T}$ method was used to calculate the relative expression level of CSLD genes (Additional file 14: Table S14) [96]. The heatmap for the qRT-PCR analysis was generated by pheatmap package (pheatmap: Pretty Heatmaps, $\mathrm{R}$ package version 1.0.8, https://CRAN.Rproject.org/package=pheatmap).

\section{Positive selection}

The detection of positive selection in cotton CSLD proteincoding genes across the phylogeny with the branch-site model was implemented in slimcodeml $[97,98]$. In this model, the branch in which we test positive selection is called the foreground branch, and all other branches on the tree are called the background branches. We assume that the $\omega$ ratio varies among codon sites, and the codon sequence is divided into four site classes. Site class 0 (with proportion $p_{0}$ ) includes codons that are highly conserved or evolve under purifying selection on all branches, with $0<\omega_{0}<1$. Site class 1 (with proportion $p_{1}$ ) includes codons that are neutral, with $\omega_{1}=1$. Codons in site classes $2 \mathrm{a}$ and $2 \mathrm{~b}$ (with proportion 1- $p_{0}-p_{1}$ ) evolve under positive selection, with $\omega_{2}>1$, but the background branches are conserved or neutral [99]. We calculated the likelihood of positive selection at each site along the cotton branches using branch-site model A (model $=2$, NSsites $=2$ ) versus the corresponding null model. To guard against codeml getting stuck in local maxima, the analysis was conducted in triplicate with varying initial $\mathrm{dN}: \mathrm{dS}[54,100] . P$ values were estimated using a chi-square distribution with one degree of freedom. Bayes empirical Bayes (BEB) was available for calculating the posterior probability for each site [99]. Sites with BEB posterior probabilities $>0.95$ were considered under positive selection. To test whether postduplication selection represented a long-term shift in selective pressure or the evolution of functional differentiation, we performed branch-site model (model $=2$, NSsites $=2$ ) analyses at the cotton clade level, considering all branches following the duplication event as the foreground and the remaining branches as background. We additionally corrected for multiple tests using the Bonferroni correction.

\section{Structural modeling}

The secondary structure and TMHs of GrCSLD1 were predicted using the DSS algorithm of PyMOL (The PyMOL Molecular Graphics System, Version 1.7, Schrodinger, LLC) and MEMSAT [100, 101], respectively.

Computational methods for predicting three-dimensional protein structures can generally be divided into two categories, template-based (comparative and threading modeling) and template-free modeling (ab initio modeling), with some composite protocols combining aspects of 
both [102, 103]. To obtain a refined three-dimensional structure of GrCSLD1, prediction was preformed using ITASSER [104, 105], Phyre2 [106] and Robetta [107, 108]. Because Robetta uses the Ginzu method to parse the input protein sequences into domains, builds models for domains with sequence homology to proteins of PDB using comparative modeling, and models for domains without a detectable PDB homolog using the Rosetta ab initio protocol, the structure of GrCSLD1 was broken up into two putative domains, which were modeled separately. The domain models of Rosetta were evaluated using the DOPE functions of MODELER [109], Verfity3D [110], ProSA [111]. The top-scoring models of two domains were recombined together using the hybridizeMove function of RosettaCM. Candidate models of the full-length GrCSLD1 were again assessed using the DOPE functions of MODELER, Verfity3D, and ProSA. We used the TM-align structural alignment program to match the top-scoring model to the structure of BcsA [57]. The TM-score has a value in $(0,1]$, and a score higher than 0.5 indicates that two structures share the same fold in SCOP/CATH [58].

\section{Additional files}

Additional file 1: Table S1. Chromosomal locus ID and length of CSLD proteins in other plants. (XLSX $16 \mathrm{~kb}$ )

Additional file 2: The sequences of CSLD proteins in all plants. (FASTA $134 \mathrm{~kb}$ )

Additional file 3: Table S2. Model selection using ProtTest. (DOCX $23 \mathrm{~kb}$ ) Additional file 4: Table S3. Comparison of ML and Bayesian trees based on three alignments (Kalign, Mafft and Muscle) using Ktreedist. (DOCX $33 \mathrm{~kb}$ )

Additional file 5: Figure S1. The different topologies of cotton CSLD trees reconstructed from ML and Bayesian based on three alignments and the elision strategy. Support values are shown for A. thaliana-cotton and cotton CSLD nodes using different color circles as bootstrap proportions/SH-like aLRT scores/Bayesian posterior probabilities. The cotton CSLD protein clades are indicated by different colors. "Other CSLD" indicates the CSLD proteins from other plant species. (TIFF $2007 \mathrm{~kb}$ )

Additional file 6: Table S10, 11 and 12. The CESA/CSL gene count data from HTseq-count in cotton. (XLSX $31 \mathrm{~kb}$ )

Additional file 7: Table S13. Primers of the GhCSLD genes used for qRT-PCR analysis. (XLSX $9 \mathrm{~kb}$ )

Additional file 8: Figure S3. Diagram of transmembrane helices (TMHs) and the cytosolic loop in GrCSLD1. The labels within the cytosolic loop and TMHs (1-8) show the approximate locations of the four conserved motifs (black), P-CR (purple), CSR (blue), and amino acid residues under positive selection (red). (TIFF $6688 \mathrm{~kb}$ )

Additional file 9: Figure S4. Multiple sequence alignments of GrCSLD1, GhCESA1, BCSA and ATCSLD1. The secondary structure of GrCSLD1 was calculated using the DSS algorithm of PyMOL. The violet cylinders, yellow arrows, and black lines indicate the a-helices, $\beta$-strand and coil of GrCSLD1; the red rectangles and yellow rectangles indicate the a-helices and $\beta$-strand of GhCESA1, and the red lines and yellow lines indicate the a-helices and $\beta$-strand of BCSA. The plant-conserved region (P-CR) and class-specific region (CSR) are highlighted with blue and green lines. Large red letters indicate sites of episodic positive selection in GrCSLD1. (TIFF $4834 \mathrm{~kb}$ )

Additional file 10: Table S15. Model validation scores of the full-length GrCSLD1 protein. (DOCX $22 \mathrm{~kb}$ )
Additional file 11: Table S4, $\mathbf{5}$ and $\mathbf{6}$. The source of transcriptome data from G. hirsutum, G. arboreum and G. raimondii. (XLSX $12 \mathrm{~kb}$ )

Additional file 12: Table S7, 8 and 9. The express profiles of CESA/CSL gene superfamily with normalization and variance stabilizing transformation using DESeq in cotton. (XLSX $49 \mathrm{~kb}$ )

Additional file 13: Figrue S2. Phylogenetic analysis of the CESA/CSL proteins in cotton and A. thaliana. The phylogenetic tree was inferred using maximum likelihood. Support values are shown for key nodes as bootstrap proportions. (TIFF $2921 \mathrm{~kb}$ )

Additional file 14: Table S14. The relative expression level of CSLD genes of $G$. hirsutum by comparative $2^{-\Delta \Delta C T}$ method using qRT-PCR. (XLSX $10 \mathrm{~kb})$

\section{Abbreviations}

A\&G: Androecium \& gynoecium; BEB: Bayes empirical Bayes; CESA: Cellulose synthase; CSL: Cellulose synthase-like; CSLD: Cellulose synthase-like D; CSR: Class-specific region; GT2: Glycosyltransferases family 2; IF: Interface; irx: irregular xylem; ML: Maximum likelihood; MSA: Multiple sequence alignment; MYA: Million years ago; P-CR: Plant-conserved region; RMSD: Root mean square deviation; TMH: Transmembrane helix; WGD: Whole-genome duplication

\section{Acknowledgments}

The authors would like to thank Dr. Wu Li and Miss Wenfeng Fu (Industrial Crop Research Institute, Henan Academy of Agricultural Sciences) for assistance with qRT-PCR analysis.

\section{Funding}

This work was supported by the Scientific and Technological Project of Henan Province (No. 152102110130).

\section{Availability of data and materials}

The data sets supporting the results of this article are included within the article and its additional files. The materials described in the article are freely available upon request to any scientist wishing to use them for non-commercial purposes.

\section{Authors' contributions}

YPL and TGY conceived and designed the study. YPL performed the bioinformatics experiments and wrote the manuscript. DDD and YH collected the sequence data and performed the GRT-PCR analysis. XYG and HXG collected tissue and performed RNA extraction. All authors read and approved the final manuscript.

Ethics approval and consent to participate

Not applicable.

\section{Consent for publication}

Not applicable.

\section{Competing interests}

The authors declare that they have no competing interests.

\section{Publisher's Note}

Springer Nature remains neutral with regard to jurisdictional claims in published maps and institutional affiliations.

\section{Author details}

${ }^{1}$ Industrial Crop Research Institute, Henan Academy of Agricultural Sciences, No. 116, Huayuan Road, Zhengzhou 450002, China. ${ }^{2}$ Scientific Observing and Experimental Station of Crop Cultivation in Central Plain, Ministry of Agriculture, No. 116, Huayuan Road, Zhengzhou 450002, China.

Received: 13 December 2016 Accepted: 25 June 2017

Published online: 10 July 2017

References

1. Taylor NG. Cellulose biosynthesis and deposition in higher plants. New Phytol. 2008;178(2):239-52. doi:10.1111/j.1469-8137.2008.02385.x. 
2. McFarlane HE, Döring A, Persson S. The cell biology of cellulose synthesis. Annu Rev Plant Biol. 2014;65(1):69-94. doi:10.1146/annurev-arplant-050213-040240.

3. Somerville C, Bauer S, Brininstool G, Facette M, Hamann T, Milne J, et al. Toward a systems approach to understanding plant cell walls. Science. 2004;306(5705):2206-11. doi:10.1126/science.1102765.

4. Vanholme R, Demedts B, Morreel K, Ralph J, Boerjan W. Lignin biosynthesis and structure. Plant Physiol. 2010;153(3):895-905. doi:10.1104/pp.110.155119.

5. Somerville C. Cellulose synthesis in higher plants. Annu Rev Cell Dev Biol. 2006;22(1):53-78. doi:10.1146/annurev.cellbio.22.022206.160206.

6. Cosgrove DJ. Re-constructing our models of cellulose and primary cell wall assembly. Curr Opin Plant Biol. 2014;22:122-31. doi:10.1016/j.pbi.2014.11.001.

7. Wang Y, Fan C, Hu H, Li Y, Sun D, Wang Y, et al. Genetic modification of plant cell walls to enhance biomass yield and biofuel production in bioenergy crops. Biotechnol Adv. 2016;34(5):997-1017. doi:10.1016/j. biotechadv.2016.06.001.

8. Burton RA, Fincher GB. Plant cell wall engineering: applications in biofuel production and improved human health. Curr Opin Biotechnol. 2014;26:79-84. doi:10.1016/j.copbio.2013.10.007

9. Cantarel BL, Coutinho PM, Rancurel C, Bernard T, Lombard V, Henrissat B. The carbohydrate-active enzymes database (CAZy): an expert resource for glycogenomics. Nucleic Acids Res. 2009;37(suppl 1):D233-D38. doi:10.1093/ nar/gkn663.

10. Yin Y, Johns MA, Cao H, Rupani M. A survey of plant and algal genomes and transcriptomes reveals new insights into the evolution and function of the cellulose synthase superfamily. BMC Genomics. 2014;15(1):1-15. doi:10.1186/1471-2164-15-260.

11. Yin $Y$, Huang J, Xu Y. The cellulose synthase superfamily in fully sequenced plants and algae. BMC Plant Biol. 2009;9:1-14. doi:10.1186/1471-2229-9-99.

12. Richmond TA, Somerville CR. The cellulose synthase superfamily. Plant Physiol. 2000;124(2):495-8. doi:10.1104/pp.124.2.495.

13. Wang L, Guo K, Li Y, Tu Y, Hu H, Wang B, et al. Expression profiling and integrative analysis of the CESA/CSL superfamily in rice. BMC Plant Biol. 2010;10(1):1-16. doi:10.1186/1471-2229-10-282.

14. Hazen SP, Scott-Craig JS, Walton JD. Cellulose synthase-like genes of rice. Plant Physiol. 2002;128(2):336-40. doi:10.1104/pp.010875.

15. Pear JR, Kawagoe Y, Schreckengost WE, Delmer DP, Stalker DM. Higher plants contain homologs of the bacterial celA genes encoding the catalytic subunit of cellulose synthase. Proc Natl Acad Sci U S A. 1996;93(22):12637-42.

16. Desprez T, Juraniec M, Crowell EF, Jouy H, Pochylova Z, Parcy F, et al. Organization of cellulose synthase complexes involved in primary cell wall synthesis in Arabidopsis thaliana. Proc Natl Acad Sci U S A. 2007;104(39): 15572-7. doi:10.1073/pnas.0706569104.

17. Persson S, Paredez A, Carroll A, Palsdottir H, Doblin M, Poindexter P, et al. Genetic evidence for three unique components in primary cell-wall cellulose synthase complexes in Arabidopsis. Proc Natl Acad Sci U S A. 2007;104(39):15566-71. doi:10.1073/pnas.0706592104.

18. Taylor NG, Howells RM, Huttly AK, Vickers K, Turner SR. Interactions among three distinct CesA proteins essential for cellulose synthesis. Proc Natl Acad Sci U S A. 2003;100(3):1450-5. doi:10.1073/pnas.0337628100.

19. Li A, Xia T, Xu W, Chen T, Li X, Fan J, et al. An integrative analysis of four CESA isoforms specific for fiber cellulose production between Gossypium hirsutum and Gossypium barbadense. Planta. 2013;237(6):1585-97. doi:10.1007/s00425-013-1868-2.

20. Paterson AH, Wendel JF, Gundlach H, Guo H, Jenkins J, Jin D, et al. Repeated polyploidization of Gossypium genomes and the evolution of spinnable cotton fibres. Nature. 2012;492(7429):423-7. doi:10.1038/nature11798.

21. Tuttle JR, Nah G, Duke MV, Alexander DC, Guan X, Song Q, et al. Metabolomic and transcriptomic insights into how cotton fiber transitions to secondary wall synthesis, represses lignification, and prolongs elongation. BMC Genomics. 2015;16(1):1-28. doi:10.1186/s12864-015-1708-9.

22. Zhang $T$, Hu $Y$, Jiang W, Fang L, Guan X, Chen J, et al. Sequencing of allotetraploid cotton (Gossypium hirsutum L. acc. TM-1) provides a resource for fiber improvement. Nat Biotech. 2015;33(5):531-7. doi:10.1038/nbt.3207

23. Yuan D, Tang Z, Wang M, Gao W, Tu L, Jin X, et al. The genome sequence of Sea-Island cotton (Gossypium barbadense) provides insights into the allopolyploidization and development of superior spinnable fibres. Sci Rep. 2015;5:17662. doi:10.1038/srep17662.

24. Dhugga KS, Barreiro R, Whitten B, Stecca K, Hazebroek J, Randhawa GS, et al. Guar seed B-mannan synthase is a member of the cellulose synthase super gene family. Science. 2004;303(5656):363-6. doi:10.1126/ science.1090908.
25. Liepman AH, Wilkerson CG, Keegstra K. Expression of cellulose synthase-like (Csl) genes in insect cells reveals that Cs/A family members encode mannan synthases. Proc Natl Acad Sci U S A. 2005;102(6):2221-6. doi:10.1073/pnas.0409179102.

26. Cocuron J-C, Lerouxel O, Drakakaki G, Alonso AP, Liepman AH, Keegstra K, et al. A gene from the cellulose synthase-like $C$ family encodes a $\beta-1,4$ glucan synthase. Proc Natl Acad Sci U S A. 2007;104(20):8550-5. doi:10.1073/pnas.0703133104

27. Doblin MS, Pettolino FA, Wilson SM, Campbell R, Burton RA, Fincher GB, et al. A barley cellulose synthase-like CSLH gene mediates $(1,3 ; 1,4)-\beta$ - $d$ glucan synthesis in transgenic Arabidopsis. Proc Natl Acad Sci U S A. 2009;106(14):5996-6001. doi:10.1073/pnas.0902019106.

28. Burton RA, Wilson SM, Hrmova M, Harvey AJ, Shirley NJ, Medhurst A, et al. Cellulose synthase-like CsIF genes mediate the synthesis of cell wall $(1,3 ; 1,4)$ beta-D-glucans. Science. 2006;311(5769):1940-2. doi:10.1126/science.1122975.

29. Park S, Szumlanski AL, Gu F, Guo F, Nielsen E. A role for CSLD3 during cell-wall synthesis in apical plasma membranes of tip-growing root-hair cells. Nat Cell Biol. 2011;13(8):973-80. doi:10.1038/ncb2294.

30. Wang W, Wang L, Chen C, Xiong G, Tan X-Y, Yang K-Z, et al. Arabidopsis CSLD1 and CSLD4 are required for cellulose deposition and normal growth of pollen tubes. J Exp Bot. 2011. doi:10.1093/jxb/err221.

31. Yin L, Verhertbruggen $Y$, Oikawa A, Manisseri C, Knierim B, Prak L, et al. The cooperative activities of CSLD2, CSLD3, and CSLD5 are required for normal Arabidopsis development. Mol Plant. 2011;4(6):1024-37. http://dx.doi.org/10. 1093/mp/ssr026

32. Favery B, Ryan E, Foreman J, Linstead P, Boudonck K, Steer M, et al. KOJAK encodes a cellulose synthase-like protein required for root hair cell morphogenesis in Arabidopsis. Genes Dev. 2001;15(1):79-89. doi:10.1101/gad.188801.

33. Wang X, Cnops G, Vanderhaeghen R, De Block S, Van Montagu M, Van Lijsebettens M. AtCSLD3, a cellulose synthase-like gene important for root hair growth in Arabidopsis. Plant Physiol. 2001;126(2):575-86. doi:10.1104/pp.126.2.575.

34. Bernal AJ, Yoo C-M, Mutwil M, Jensen JK, Hou G, Blaukopf C, et al. Functional analysis of the cellulose synthase-like genes CSLD1, CSLD2, and CSLD4 in tip-growing Arabidopsis cells. Plant Physiol. 2008;148(3):1238-53. doi:10.1104/pp.108.121939

35. Yoo C-M, Quan L, Blancaflor EB. Divergence and redundancy in CSLD2 and CSLD3 function during Arabidopsis thaliana root hair and female gametophyte development. Front Plant Sci. 2012; 3. doi:10.3389/fpls.2012.00111.

36. Bernal AJ, Jensen JK, Harholt J, Sørensen S, Moller I, Blaukopf C, et al. Disruption of ATCSLD5 results in reduced growth, reduced xylan and homogalacturonan synthase activity and altered xylan occurrence in Arabidopsis. Plant J. 2007;52(5):791-802. doi:10.1111/j.1365-313X.2007.03281.X.

37. Gu F, Bringmann M, Combs JR, Yang J, Bergmann DC, Nielsen E. Arabidopsis CSLD5 functions in cell plate formation in a cell cycle-dependent manner. Plant Cell. 2016;28(7):1722-37. doi:10.1105/tpc.16.00203.

38. Kim CM, Park SH, Je BI, Park SH, Park SJ, Piao HL, et al. OsCSLD1, a cellulose synthase-like D1 gene, is required for root hair morphogenesis in Rice. Plant Physiol. 2007;143(3):1220-30. doi:10.1104/pp.106.091546.

39. Luan W, Liu Y, Zhang F, Song Y, Wang Z, Peng Y, et al. OsCD1 encodes a putative member of the cellulose synthase-like $D$ sub-family and is essential for rice plant architecture and growth. Plant Biotechnol J. 2011;9(4):513-24. doi:10.1111/j.1467-7652.2010.00570.x.

40. Wu C, Fu Y, Hu G, Si H, Cheng S, Liu W. Isolation and characterization of a rice mutant with narrow and rolled leaves. Planta. 2010;232(2):313-24. doi:10.1007/s00425-010-1180-3.

41. Hu J, Zhu L, Zeng D, Gao Z, Guo L, Fang Y, et al. Identification and characterization of NARROW AND ROLLED LEAF 1, a novel gene regulating leaf morphology and plant architecture in rice. Plant Mol Biol. 2010;73(3): 283-92. doi:10.1007/s11103-010-9614-7.

42. Hunter $C$, Kirienko DH, Sylvester AW, Peter GF, McCarty DR, Koch KE. Cellulose synthase-like D1 is integral to normal cell division, expansion, and leaf development in maize. Plant Physiol. 2012;158(2):708-24. doi:10.1104/pp.111.188466.

43. Wang K, Wang Z, Li F, Ye W, Wang J, Song G, et al. The draft genome of a diploid cotton Gossypium raimondii. Nat Genet. 2012;44(10):1098-103. doi:10.1038/ng.2371.

44. Wendel JF, Flagel LE, Adams KL. Jeans, genes, and genomes: cotton as a model for studying polyploidy. In: Soltis SP, Soltis ED, editors. Polyploidy and genome evolution. Berlin: Springer Berlin Heidelberg; 2012. p. 181-207. doi:10.1007/978-3-642-31442-1_10. 
45. Li F, Fan G, Lu C, Xiao G, Zou C, Kohel RJ, et al. Genome sequence of cultivated upland cotton (Gossypium hirsutum TM-1) provides insights into genome evolution. Nat Biotech. 2015;33(5):524-30. doi:10.1038/nbt.3208.

46. Qin Y-M, Zhu Y-X. How cotton fibers elongate: a tale of linear cell-growth mode. Curr Opin Plant Biol. 2011;14(1):106-11. doi:10.1016/j.pbi.2010.09.010.

47. Hernandez-Gomez MC, Runavot J-L, Guo X, Bourot S, Benians TAS, Willats WGT, et al. Heteromannan and heteroxylan cell wall polysaccharides display different dynamics during the elongation and secondary cell wall deposition phases of cotton fiber cell development. Plant Cell Physiol. 2015; 56(9):1786-97. doi:10.1093/pcp/pcv101.

48. Li F, Fan G, Wang K, Sun F, Yuan Y, Song G, et al. Genome sequence of the cultivated cotton Gossypium arboreum. Nat Genet. 2014;46(6):567-72. doi:10.1038/ng.2987

49. Ronquist F, Teslenko M, van der Mark P, Ayres DL, Darling A, Höhna S, et al. MrBayes 3.2: efficient bayesian phylogenetic inference and model choice across a large model space. Syst Biol. 2012;61(3):539-42. doi:10.1093/sysbio/sys029.

50. Kelley JL, Swanson WJ. Positive selection in the human genome: from genome scans to biological significance. Annu Rev Genomics Hum Genet. 2008;9(1):143-60. doi:10.1146/annurev.genom.9.081307.164411.

51. Yang Z, Bielawski JP. Statistical methods for detecting molecular adaptation. Trends Ecol Evol. 2000;15(12):496-503. doi:10.1016/S0169-5347(00)01994-7.

52. Bishop JG, Ripoll DR, Bashir S, Damasceno CMB, Seeds JD, Rose JKC. Selection on glycine $\beta-1,3$-endoglucanase genes selection on glycine $\beta-1,3-$ endoglucanase genes differentially inhibited by a Phytophthora glucanase inhibitor protein. Genetics. 2005;169(2):1009-19. doi:10.1534/genetics.103.025098.

53. Innan H, Kondrashov F. The evolution of gene duplications: classifying and distinguishing between models. Nat Rev Genet. 2010;11(2):97-108. doi:10.1038/nrg2689.

54. Schwerdt JG, MacKenzie K, Wright F, Oehme D, Wagner JM, Harvey AJ, et al. Evolutionary dynamics of the cellulose synthase gene superfamily in grasses. Plant Physiol. 2015;168(3):968-83. doi:10.1104/pp.15.00140.

55. Garvin MR, Bielawski JP, Gharrett AJ. Positive Darwinian selection in the piston that powers proton pumps in complex I of the mitochondria of pacific salmon. PLoS One. 2011;6(9):e24127. doi:10.1371/journal.pone.0024127.

56. Chen K, Durand D, Farach-Colton M. NOTUNG:: A program for dating gene duplications and optimizing gene family Trees. J Comput Biol 2000; 7(3-4): 429-447. doi:10.1089/106652700750050871.

57. Morgan JLW, Strumillo J, Zimmer J. Crystallographic snapshot of cellulose synthesis and membrane translocation. Nature. 2013;493(7431): 181-6. doi:10.1038/nature11744.

58. Zhang Y, Skolnick J. TM-align: a protein structure alignment algorithm based on the TM-score. Nucleic Acids Res. 2005;33(7):2302-9. doi:10.1093/nar/gki524.

59. Slabaugh E, Davis JK, Haigler CH, Yingling YG, Zimmer J. Cellulose synthases: new insights from crystallography and modeling. Trends Plant Sci. 2014; 19(2):99-106. doi:10.1016/j.tplants.2013.09.009.

60. Lairson LL, Henrissat B, Davies GJ, Withers SG. Glycosyltransferases: structures, functions, and mechanisms. Annu Rev Biochem. 2008;77(1):521-55. doi:10.1146/annurev.biochem.76.061005.092322.

61. Sethaphong L, Haigler CH, Kubicki JD, Zimmer J, Bonetta D, DeBolt S, et al. Tertiary model of a plant cellulose synthase. Proc Natl Acad Sci U S A. 2013; 110(18):7512-7. doi:10.1073/pnas.1301027110.

62. Pettersen EF, Goddard TD, Huang CC, Couch GS, Greenblatt DM, Meng EC, et al. UCSF chimera-a visualization system for exploratory research and analysis. J Comput Chem. 2004;25(13):1605-12. doi:10.1002/jcc.20084.

63. Soderlund C, Bomhoff M, Nelson WM. SyMAP v3.4: a turnkey synteny system with application to plant genomes. Nucleic Acids Res. 2011;39(10): e68. doi:10.1093/nar/gkr123.

64. Tang H, Wang X, Bowers JE, Ming R, Alam M, Paterson AH. Unraveling ancient hexaploidy through multiply-aligned angiosperm gene maps. Genome Res. 2008;18(12):1944-54. doi:10.1101/gr.080978.108.

65. Ogden $\mathrm{TH}$, Rosenberg MS. Multiple sequence alignment accuracy and phylogenetic inference. Syst Biol. 2006;55(2):314-28. doi:10.1080/ 10635150500541730.

66. Castresana J. Selection of conserved blocks from multiple alignments for their use in phylogenetic analysis. Mol Biol Evol. 2000;17(4):540-52. doi:10. 1093/oxfordjournals.molbev.a026334.

67. Felsenstein J. Evolutionary trees from DNA sequences: a maximum likelihood approach. J Mol Evol. 1981;17(6):368-76. doi:10.1007/bf01734359.

68. Huelsenbeck JP, Ronquist F, Nielsen R, Bollback JP. Bayesian inference of phylogeny and its impact on evolutionary biology. Science. 2001;294(5550): 2310-4. doi:10.1126/science.1065889.
69. Maddison W. Reconstructing character evolution on polytomous cladograms. Cladistics. 1989;5(4):365-77. doi:10.1111/j.1096-0031.1989.tb00569.x.

70. Guo K, Zou W, Feng Y, Zhang M, Zhang J, Tu F, et al. An integrated genomic and metabolomic framework for cell wall biology in rice. BMC Genomics. 2014;15(1):596. doi:10.1186/1471-2164-15-596.

71. Li A, Wang R, Li X, Liu M, Fan J, Guo K, et al. Proteomic profiling of cellulaseaid-extracted membrane proteins for functional identification of cellulose synthase complexes and their potential associated-components in cotton fibers. Sci Rep. 2016;6:26356. doi:10.1038/srep26356.

72. Altschul SF, Madden TL, Schäffer AA, Zhang J, Zhang Z, Miller W, et al. Gapped BLAST and PSI-BLAST: a new generation of protein database search programs. Nucleic Acids Res. 1997;25(17):3389-402. doi:10.1093/nar/25.17.3389.

73. Goodstein DM, Shu S, Howson R, Neupane R, Hayes RD, Fazo J, et al. Phytozome: a comparative platform for green plant genomics. Nucleic Acids Res. 2012;40(D1):D1178-D86. doi:10.1093/nar/gkr944.

74. Letunic I, Doerks T, Bork P. SMART: recent updates, new developments and status in 2015. Nucleic Acids Res. 2015;43(D1):D257-D60. doi:10.1093/nar/gku949.

75. Mitchell A, Chang H-Y, Daugherty L, Fraser M, Hunter S, Lopez R, et al. The InterPro protein families database: the classification resource after 15 years. Nucleic Acids Res. 2015;43(D1):D213-D21. doi:10.1093/nar/gku1243.

76. Marchler-Bauer A, Derbyshire MK, Gonzales NR, Lu S, Chitsaz F, Geer LY, et al. CDD: NCBI's conserved domain database. Nucleic Acids Res. 2015;43(D1):D222-D26. doi:10.1093/nar/gku1221.

77. Bayer M, Milne I, Stephen G, Shaw P, Cardle L, Wright F, et al. Comparative visualization of genetic and physical maps with strudel. Bioinformatics. 2011; 27(9):1307-8. doi:10.1093/bioinformatics/btr111.

78. Lassmann T, Frings O, Sonnhammer ELL. Kalign2: high-performance multiple alignment of protein and nucleotide sequences allowing external features. Nucleic Acids Res. 2009;37(3):858-65. doi:10.1093/nar/gkn1006.

79. Katoh K, Standley DM. MAFFT multiple sequence alignment software version 7: improvements in performance and usability. Mol Biol Evol. 2013; 30(4):772-80. doi:10.1093/molbev/mst010.

80. Edgar RC. MUSCLE: multiple sequence alignment with high accuracy and high throughput. Nucleic Acids Res. 2004;32(5):1792-7. doi:10.1093/nar/gkh340.

81. Talavera G, Castresana J. Improvement of phylogenies after removing divergent and ambiguously aligned blocks from protein sequence alignments. Syst Biol. 2007;56(4):564-77. doi:10.1080/10635150701472164.

82. Wheeler WC, Gatesy J, DeSalle R. Elision: A method for accommodating multiple molecular sequence alignments with alignment-ambiguous sites. Mol Phylogen Evol. 1995;4(1):1-9. doi:10.1006/mpev.1995.1001.

83. Guindon S, Dufayard J-F, Lefort V, Anisimova M, Hordijk W, Gascuel O. New algorithms and methods to estimate maximum-likelihood phylogenies: assessing the performance of PhyML 3.0. Syst Biol. 2010;59(3):307-21. doi:10.1093/sysbio/syq010.

84. Darriba D, Taboada GL, Doallo R, Posada D. ProtTest 3: fast selection of best-fit models of protein evolution.Bioinformatics. 2011;27(8):1164-5. doi:10.1093/bioinformatics/btr088.

85. Anisimova M, Gil M, Dufayard J-F, Dessimoz C, Gascuel O. Survey of branch support methods demonstrates accuracy, power, and robustness of fast likelihood-based approximation schemes. Syst Biol. 2011;60(5):685-99. doi:10.1093/sysbio/syr041.

86. Soria-Carrasco V, Talavera G, Igea J, Castresana J. The K tree score: quantification of differences in the relative branch length and topology of phylogenetic trees. Bioinformatics. 2007;23(21):2954-6. doi:10.1093/bioinformatics/btm466.

87 Kim D, Pertea G, Trapnell C, Pimentel H, Kelley R, Salzberg SL. TopHat2: accurate alignment of transcriptomes in the presence of insertions, deletions and gene fusions. Genome Biol. 2013;14(4):R36. doi:10.1186/gb-2013-14-4-r36.

88. Li H, Handsaker B, Wysoker A, Fennell T, Ruan J, Homer N, et al. The sequence alignment/map format and SAMtools. Bioinformatics. 2009;25(16): 2078-9. doi:10.1093/bioinformatics/btp352.

89. Anders S, Pyl PT, Huber W. HTSeq - a python framework to work with high-throughput sequencing data. Bioinformatics. 2015;31(2):166-9. doi:10.1093/bioinformatics/btu638.

90. Anders S, Huber W. Differential expression analysis for sequence count data. Genome Biol. 2010;11(10):R106. doi:10.1186/gb-2010-11-10-r106.

91. Anders S, McCarthy DJ, Chen Y, Okoniewski M, Smyth GK, Huber W, et al. Count-based differential expression analysis of RNA sequencing data using $\mathrm{R}$ and bioconductor. Nat Protocols. 2013;8(9):1765-86. doi:10.1038/nprot. 2013.099 .

92. Eddy SR. Accelerated pofile HMM searches. PLoS Comp Biol. 2011;7(10): e1002195. doi:10.1371/journal.pcbi.1002195. 
93. Lamesch P, Berardini TZ, Li D, Swarbreck D, Wilks C, Sasidharan R, et al. The Arabidopsis information resource (TAIR): improved gene annotation and new tools. Nucleic Acids Res. 2012;40(D1):D1202-D10. doi:10.1093/ nar/gkr1090.

94. Fu W, Shen Y, Hao J, Wu J, Ke L, Wu C, et al. Acyl-CoA N-acyltransferase influences fertility by regulating lipid metabolism and jasmonic acid biogenesis in cotton. Sci Rep. 2015:5:11790. doi:10.1038/srep11790.

95. Li W, Zhao FA, Fang W, Xie D, Hou J, Yang X, et al. Identification of early salt stress responsive proteins in seedling roots of upland cotton (Gossypium hirsutum L.) employing iTRAQ-based proteomic technique. Front Plant Sci. 2015;6(732). doi:10.3389/fpls.2015.00732.

96. Livak KJ, Schmittgen TD. Analysis of relative gene expression data using real-time quantitative PCR and the $2^{-\Delta \Delta C T}$ method. Methods. 2001;25(4):402-8. doi:10.1006/meth.2001.1262.

97 Schabauer $H$, Valle M, Pacher C, Stockinger H, Stamatakis A, Robinson-Rechavi $M$, et al. SlimCodeML: an optimized version of CodeML for the branch-site model. In: Parallel and Distributed Processing Symposium Workshops \& PhD Forum (IPDPSW), 2012 IEEE 26th International. 2012;706-14. doi:10.1109/ IPDPSW.2012.88.

98. Yang Z. PAML 4: Phylogenetic analysis by maximum likelihood. Mol Biol Evol. 2007;24(8):1586-91. doi:10.1093/molbev/msm088.

99. Zhang J, Nielsen R, Yang Z. Evaluation of an improved branch-site likelihood method for detecting positive selection at the molecular level. Mol Biol Evol. 2005;22(12):2472-9. doi:10.1093/molbev/msi237.

100. Fletcher $W$, Yang Z. The effect of insertions, deletions, and alignment errors on the branch-site test of positive selection. Mol Biol Evol. 2010;27(10):2257-67. doi:10.1093/molbev/msq115.

101. Nugent T, Jones DT. Transmembrane protein topology prediction using support vector machines. BMC Bioinformatics. 2009;10(1):1-11. doi:10.1186/ 1471-2105-10-159.

102. Jones DT. Improving the accuracy of transmembrane protein topology prediction using evolutionary information. Bioinformatics. 2007;23(5):538-44. doi:10.1093/bioinformatics/btl677.

103. Källberg M, Wang H, Wang S, Peng J, Wang Z, Lu H, et al. Template-based protein structure modeling using the RaptorX web server. Nat Protoc. 2012; 7(8):1511-22. doi:10.1038/nprot.2012.085.

104. Roy A, Kucukural A, Zhang Y. I-TASSER: a unified platform for automated protein structure and function prediction. Nat Protoc. 2010;5(4):725-38. doi: 10.1038/nprot.2010.5.

105. Zhang Y. I-TASSER server for protein 3D structure prediction. BMC Bioinformatics. 2008;9(1):1-8. doi:10.1186/1471-2105-9-40.

106. Kelley LA, Mezulis S, Yates CM, Wass MN, Sternberg MJE. The Phyre2 web portal for protein modeling, prediction and analysis. Nat Protoc. 2015;10(6): 845-58. doi:10.1038/nprot.2015.053.

107. Song Y, DiMaio F, Wang Ray Y-R, Kim D, Miles C, Brunette TJ, et al. Highresolution comparative modeling with RosettaCM. Structure. 2013;21(10): 1735-42. doi:10.1016/j.str.2013.08.005.

108. Raman S, Vernon R, Thompson J, Tyka M, Sadreyev R, Pei J, et al. Structure prediction for CASP8 with all-atom refinement using Rosetta. Proteins. 2009; 77(S9):89-99. doi:10.1002/prot.22540.

109. Šali A, Blundell TL. Comparative protein modelling by satisfaction of spatial restraints. J Mol Biol. 1993;234(3):779-815. doi:10.1006/jmbi.1993.1626.

110. Luthy $R$, Bowie JU, Eisenberg D. Assessment of protein models with threedimensional profiles. Nature. 1992;356(6364):83-5. doi:10.1038/356083a0.

111. Wiederstein M, Sippl MJ. ProSA-web: interactive web service for the recognition of errors in three-dimensional structures of proteins. Nucleic Acids Res. 2007;35(suppl 2):W407-W10. doi:10.1093/nar/gkm290.

112. Liu W, Xie Y, Ma J, Luo X, Nie P, Zuo Z, et al. IBS: an illustrator for the presentation and visualization of biological sequences. Bioinformatics. 2015; 31(20):3359-61. doi:10.1093/bioinformatics/btv362.

\section{Submit your next manuscript to BioMed Central and we will help you at every step:}

- We accept pre-submission inquiries

- Our selector tool helps you to find the most relevant journal

- We provide round the clock customer support

- Convenient online submission

- Thorough peer review

- Inclusion in PubMed and all major indexing services

- Maximum visibility for your research

Submit your manuscript at www.biomedcentral.com/submit
C Biomed Central 